\title{
OPTIMAL CONTROL OF THE TWO-DIMENSIONAL STATIONARY NAVIER-STOKES EQUATIONS WITH MEASURE VALUED CONTROLS*
}

\author{
EDUARDO $\mathrm{CASAS}^{\dagger}$ AND KARL KUNISCH ${ }^{\ddagger}$
}

\begin{abstract}
In this paper, we consider an optimal control problem for the two-dimensional stationary Navier-Stokes system. Looking for sparsity, we take Borel measures as controls. We prove the well-posedness of the control problem and derive necessary and sufficient conditions for local optimality of the controls. Finally, under a second order condition, we prove rates of the optimal states with respect to small perturbations in the data of the control problem.
\end{abstract}

Key words. Navier-Stokes equations, Borel measures, sparsity, first and second order optimality conditions, stability

AMS subject classifications. 49J20, 49J52, 49K20, 49K40

DOI. $10.1137 / 18 \mathrm{M} 1185582$

1. Introduction. In this paper we investigate the following optimal control problem

$$
(\mathrm{P}) \min _{(\mathbf{y}, \mathbf{u}) \in \mathbf{L}^{2}(\Omega) \times \mathbf{M}(\omega)} \mathcal{J}(\mathbf{y}, \mathbf{u})=\frac{1}{2} \int_{\Omega}\left|\mathbf{y}-\mathbf{y}_{d}\right|^{2} d x+\alpha\|\mathbf{u}\|_{\mathbf{M}(\omega)},
$$

where $\mathbf{y}$ and $\mathbf{u}$ are related by the Navier-Stokes system

$$
\left\{\begin{array}{l}
-\nu \Delta \mathbf{y}+(\mathbf{y} \cdot \nabla) \mathbf{y}+\nabla \mathfrak{p}=\mathbf{f}_{0}+\chi_{\omega} \mathbf{u} \text { in } \Omega, \\
\operatorname{div} \mathbf{y}=0 \text { in } \Omega, \mathbf{y}=0 \text { on } \Gamma .
\end{array}\right.
$$

Here, $\Omega$ denotes a bounded domain in $\mathbb{R}^{2}$ with a $C^{2}$ boundary $\Gamma$, and $\omega$ is a relatively closed subset of $\Omega$. We denote $\mathbf{M}(\omega)=M(\omega) \times M(\omega)$, where $M(\omega)$ is the space of real and regular Borel measure in $\omega$. In the cost functional $\mathcal{J}$, the target $\mathbf{y}_{d} \in \mathbf{L}^{2}(\Omega)$ and the parameter $\alpha>0$ are fixed. Regarding the state equation, $\nu>0$ is the kinematic viscosity coefficient, $\chi_{\omega} \mathbf{u}$ denotes the extension of $\mathbf{u}$ by zero outside $\omega$, and $\mathbf{f}_{0}$ is a given element of $W^{-1, p}(\Omega) \times W^{-1, p}(\Omega)$, where $p \in(4 / 3,2)$ is fixed.

Let us comment about the norm in $\mathbf{M}(\omega)$ appearing in $\mathcal{J}$. First, we recall that $M(\omega)$ is a Banach space when endowed with the norm

$$
\|u\|_{M(\omega)}=\sup _{\|\phi\|_{C_{0}(\omega)} \leq 1} \int_{\omega} \phi(x) d u(x)=|u|(\omega)
$$

where

$$
C_{0}(\omega)=\{\phi \in C(\bar{\omega}): \phi(x)=0 \quad \forall x \in \partial \omega \cap \Gamma\}
$$

${ }^{*}$ Received by the editors May 4, 2018; accepted for publication (in revised form) February 8, 2019; published electronically April 9, 2019.

http://www.siam.org/journals/sicon/57-2/M118558.html

Funding: The first author was supported by the Spanish Ministerio de Economía, Industria y Competitividad under research projects MTM2014-57531-P and MTM2017-83185-P. The second author was supported by the ERC advanced grant 668998 (OCLOC) under the EU's H2020 research program.

${ }^{\dagger}$ Departamento de Matemática Aplicada y Ciencias de la Computación, E.T.S.I. Industriales y de Telecomunicación, Universidad de Cantabria, 39005 Santander, Spain (eduardo.casas@unican.es).

${ }^{\ddagger}$ Institute for Mathematics and Scientific Computing, University of Graz, Heinrichstrasse 36, A-8010 Graz, Austria (karl.kunisch@uni-graz.at). 
is a separable Banach space, and $|u|$ represents the total variation measure of $u$; see [23, page 130]. Note that $C_{0}(\omega) \neq C(\omega)$ only in the case that $\bar{\omega}$ has a nonempty intersection with the boundary of $\Omega$.

Associated to a norm ||$_{\mathbb{R}^{2}}$ in $\mathbb{R}^{2}$ we define

$$
\|\mathbf{u}\|_{\mathbf{M}(\omega)}=\left|\left(\left\|u_{1}\right\|_{M(\omega)},\left\|u_{2}\right\|_{M(\omega)}\right)\right|_{\mathbb{R}^{2}},
$$

which makes $\mathbf{M}(\omega)$ a Banach space. It is the dual space of $\mathbf{C}_{0}(\omega)=C_{0}(\omega) \times C_{0}(\omega)$.

To the best of our knowledge this is the first work that addresses the optimal control of Navier-Stokes equations with Borel measures as controls. The motivation for this approach is the search of optimal controls which are sparse, allowing controls having a support of zero Lebesgue measure. Such type of controls have been investigated for linear partial differential equations; see [1], [2], [3], [5], [9], [8], [10], [11], [19], [20]. The only work addressing the case of nonlinear partial differential equations is [4]. Different type of sparsity promoting formulations in a function space setting have been investigated. We mention the first one in the framework of partial differential equations [25] and refer to [1] for additional references.

A first difficulty in the analysis of the control problem $(\mathrm{P})$ is its well-posedness. The usual approach to prove the existence of an optimal solution is based on the coercivity of the cost functional with respect to the control, which implies boundedness of the states with respect to the controls through the state equation. For our state equation, estimates of the states in terms of the measure space norm of the controls are not available. To address this difficulty we also require coercivity of the cost functional with respect to the state variable. The choice of this functional enjoying the required coercivity is delicate. Here we want to do it in such a way that we obtain existence of solutions and at the same time a second order analysis can be carried out. For this purpose we have carefully analyzed the state equation getting estimates showing that the coercivity of the cost functional with respect to the state in $L^{p}$ for any $p \in[1, \infty)$ is sufficient for existence. The choice $p=2$ allows us to prove existence of a solution and to address the second order analysis. In particular, this permits us to treat the classical tracking cost functional.

Due to the nonuniqueness of solution of the Navier-Stokes systems, an assumption guaranteeing local uniqueness of the state equation around the optimal controls is needed to derive first and second order optimality conditions. In the literature this assumption relates to the smallness of the controls with respect to the kinematic viscosity. Our regularity condition is more general in the sense that it is satisfied whenever the smallness assumption on the the controls is fulfilled. Since the norm of the measures appears in the cost functional, the second order optimality conditions does not fit in the classical second order analysis. We provide a second order condition that is finally used to prove stability of the optimal states with respect to perturbations of the data in the formulation of the control problem.

For related papers addressing the control of the stationary Navier-Stokes system the reader is referred to [6], [12], [13], [17], [26].

All along this paper the following spaces will be considered.

- $\mathbf{H}_{0}^{1}(\Omega)=H_{0}^{1}(\Omega) \times H_{0}^{1}(\Omega)$

- $\mathbf{W}_{0}^{1, p}(\Omega)=W_{0}^{1, p}(\Omega) \times W_{0}^{1, p}(\Omega)$

- $\mathbf{V}=\left\{\mathbf{y} \in \mathbf{H}_{0}^{1}(\Omega): \operatorname{div} \mathbf{y}=0\right.$ in $\left.\Omega\right\}$

- $\mathbf{W}_{p}=\left\{\mathbf{y} \in \mathbf{W}_{0}^{1, p}(\Omega): \operatorname{div} \mathbf{y}=0\right.$ in $\left.\Omega\right\}$

- $L^{p}(\Omega) / \mathbb{R}=\left\{\phi \in L^{p}(\Omega): \int_{\Omega} \phi(x) d x=0\right\}$

Copyright (c) by SIAM. Unauthorized reproduction of this article is prohibited. 
2. Analysis of the state equation. In this section, we will study the existence and regularity of solutions of (1.1), as well as the differentiable dependence with respect to the right-hand side. The analysis of existence of solution will be carried out for an arbitrary $p \in(1, \infty)$ and $\mathbf{f} \in \mathbf{W}^{-1, p}(\Omega)$. We recall that $\mathbf{M}(\omega)$ is compactly embedded in $\mathbf{W}^{-1, p}(\Omega)$ for every $1<p<2$. Later, in the analysis of $(\mathrm{P})$ we will fix $p$ in $(4 / 3,2)$.

Let us start by giving the definition of solution of (1.1). To this end we first observe that $(\mathbf{y} \cdot \nabla) \mathbf{y} \in \mathbf{L}^{1}(\Omega)$ for all $\mathbf{y} \in \mathbf{W}_{0}^{1, p}(\Omega)$ with $p \geq 4 / 3$. This inclusion is a consequence of the embedding $\mathbf{W}_{0}^{1, p}(\Omega) \subset \mathbf{L}^{p^{\prime}}(\Omega)$ for $p \geq 4 / 3$. However, the integrability can fail for $p<4 / 3$. Therefore, the function $(\mathbf{y} \cdot \nabla) \mathbf{y}$ is not a distribution in such a case. To overcome this difficulty we formulate the state equation in the following way

$$
\left\{\begin{array}{l}
-\nu \Delta \mathbf{y}+\operatorname{div}(\mathbf{y} \otimes \mathbf{y})+\nabla \mathfrak{p}=\mathbf{f} \text { in } \Omega, \\
\operatorname{div} \mathbf{y}=0 \text { in } \Omega, \mathbf{y}=0 \text { on } \Gamma .
\end{array}\right.
$$

Due to the fact that $\operatorname{div} \mathbf{y}=0$ in $\Omega$, we have $\operatorname{div}(\mathbf{y} \otimes \mathbf{y})=(\mathbf{y} \cdot \nabla) \mathbf{y}$ for every $\mathbf{y} \in$ $\mathbf{W}_{0}^{1, p}(\Omega)$ with $p \geq 4 / 3$. If $(\mathbf{y} \cdot \nabla) \mathbf{z} \in \mathbf{L}^{1}(\Omega)$ we write indistinctly $\operatorname{div}(\mathbf{y} \otimes \mathbf{z})$ or $(\mathbf{y} \cdot \nabla) \mathbf{z}$.

Definition 2.1. Given $\mathbf{f} \in \mathbf{W}^{-1, p}(\Omega)$ with $1<p<\infty$, we say that $(\mathbf{y}, \mathfrak{p}) \in$ $\mathbf{W}_{0}^{1, p}(\Omega) \times L^{p}(\Omega) / \mathbb{R}$ is a solution of (2.1) if the partial differential equations of $(2.1)$ are satisfied in the distribution sense in $\Omega$. This is equivalent to the following variational identities:

$$
\left\{\begin{array}{l}
\int_{\Omega}(\nabla \mathbf{y} \nabla \mathbf{z}-(\mathbf{y} \cdot \nabla) \mathbf{z} \mathbf{y}) d x-\int_{\Omega} \mathfrak{p} \operatorname{div} \mathbf{z} d x=\langle\mathbf{f}, \mathbf{z}\rangle \quad \forall \mathbf{z} \in \mathbf{W}_{0}^{1, p^{\prime}}(\Omega), \\
\int_{\Omega} \mathfrak{q} \operatorname{div} \mathbf{y} d x=0 \quad \forall \mathfrak{q} \in L^{p^{\prime}}(\Omega) / \mathbb{R} .
\end{array}\right.
$$

Let us observe that $\mathbf{W}_{0}^{1, p}(\Omega) \subset \mathbf{L}^{2 p}(\Omega)$, hence $(\mathbf{y} \cdot \nabla) \mathbf{z} \mathbf{y} \in \mathbf{L}^{1}(\Omega)$ for every $\mathbf{z} \in \mathbf{W}_{0}^{1, p^{\prime}}(\Omega)$. Thus, the above integrals are well defined.

Remark 2.2. From (2.2) we deduce that $\mathbf{y} \in \mathbf{W}_{p}$ and

$$
\int_{\Omega}(\nabla \mathbf{y} \nabla \mathbf{z}-(\mathbf{y} \cdot \nabla) \mathbf{z} \mathbf{y}) d x=\langle\mathbf{f}, \mathbf{z}\rangle \quad \forall \mathbf{z} \in \mathbf{W}_{p^{\prime}} .
$$

Conversely, if $\mathbf{y} \in \mathbf{W}_{p}$ satisfies (2.3), then de Rham's theorem implies the existence of $\mathfrak{p} \in L^{p}(\Omega) / \mathbb{R}$ such that the first identity of (2.2) holds.

TheOREM 2.3. For every $\mathbf{f} \in \mathbf{W}^{-1, p}(\Omega)$ there exists at least one solution $(\mathbf{y}, \mathfrak{p}) \in$ $\mathbf{W}_{p} \times L^{p}(\Omega) / \mathbb{R}$ of (2.1). Furthermore, there exist constants $C_{p}$ and $M_{p}$ such that for any solution $(\mathbf{y}, \mathfrak{p})$ we have

$$
\begin{aligned}
& \|\mathbf{y}\|_{\mathbf{W}_{0}^{1, p}(\Omega)}+\|\mathfrak{p}\|_{L^{p}(\Omega)} \leq C_{p}\|\mathbf{f}\|_{\mathbf{W}^{-1, p}(\Omega)}\left(1+\|\mathbf{f}\|_{\mathbf{W}^{-1, p}(\Omega)}\right) \quad \text { if } p>2, \\
& \|\mathbf{y}\|_{\mathbf{W}_{0}^{1, p}(\Omega)}+\|\mathfrak{p}\|_{L^{p}(\Omega)} \leq C_{p}\left(\|\mathbf{f}\|_{\mathbf{W}^{-1, p}(\Omega)}+\|\mathbf{y}\|_{\mathbf{L}^{2 p}(\Omega)}^{2}\right) \quad \text { if } p<2, \\
& \|\mathbf{y}\|_{\mathbf{W}_{0}^{1, p}(\Omega)}+\|\mathfrak{p}\|_{L^{p}(\Omega)} \leq M_{p}\|\mathbf{f}\|_{\mathbf{W}^{-1, p}(\Omega)}\left(1+\|\mathbf{y}\|_{\mathbf{L}^{4}(\Omega)}\right) \quad \text { if } \frac{4}{3} \leq p<2 .
\end{aligned}
$$

Copyright $@$ by SIAM. Unauthorized reproduction of this article is prohibited. 
Proof. The existence of a solution is well known from [18, 24]. Let us prove the estimates $(2.4)-(2.6)$. For $p=2$ the a priori estimate is well known to be

$$
\|\mathbf{y}\|_{\mathbf{H}_{0}^{1}(\Omega)} \leq C_{2}\|\mathbf{f}\|_{\mathbf{H}^{-1}(\Omega)} .
$$

It uses the conservative property of the nonlinearity. Let us then turn to the case $p>2$ and write (2.1) in the form

$$
\left\{\begin{array}{l}
-\nu \Delta \mathbf{y}+\nabla \mathfrak{p}=\mathbf{g} \text { in } \Omega, \\
\operatorname{div} \mathbf{y}=0 \text { in } \Omega, \mathbf{y}=0 \text { on } \Gamma,
\end{array}\right.
$$

where $\mathbf{g}=\mathbf{f}-\operatorname{div}(\mathbf{y} \otimes \mathbf{y})$. We aim to apply the well known estimates for the Stokes problem [15, Theorem IV.6.1]

$$
\|\mathbf{y}\|_{\mathbf{W}_{0}^{1, p}(\Omega)}+\|\mathfrak{p}\|_{L^{p}(\Omega)} \leq C\|\mathbf{g}\|_{\mathbf{W}^{-1, p}(\Omega)} .
$$

In order to use this estimate to deduce (2.4) we need to prove that $\operatorname{div}(\mathbf{y} \otimes \mathbf{y}) \in$ $\mathbf{W}^{-1, p}(\Omega)$ and to get an appropriate estimate for it. For this purpose we introduce the trilinear form

$$
b(\mathbf{y}, \mathbf{z}, \mathbf{w})=\int_{\Omega}(\mathbf{y} \cdot \nabla) \mathbf{z} \mathbf{w} d x=\sum_{i, j=1}^{2} \int_{\Omega} y_{i} \partial_{x_{i}} z_{j} w_{j} d x=-\langle\operatorname{div}(\mathbf{y} \otimes \mathbf{w}), \mathbf{z}\rangle .
$$

We have for $\mathbf{y} \in \mathbf{W}_{0}^{1, p}(\Omega)$ and $\mathbf{z} \in \mathbf{W}_{0}^{1, p^{\prime}}(\Omega)$

$$
|b(\mathbf{y}, \mathbf{z}, \mathbf{y})| \leq \int_{\Omega}|\mathbf{y}|^{2}|\nabla \mathbf{z}| d x \leq\|\mathbf{y}\|_{\mathbf{L}^{2 p}(\Omega)}^{2}\|\mathbf{z}\|_{\mathbf{W}_{0}^{1, p^{\prime}}(\Omega)},
$$

which implies that $\operatorname{div}(\mathbf{y} \otimes \mathbf{y}) \in \mathbf{W}^{-1, p}(\Omega)$ and

$$
\|\operatorname{div}(\mathbf{y} \otimes \mathbf{y})\|_{\mathbf{W}^{-1, p}(\Omega)} \leq\|\mathbf{y}\|_{\mathbf{L}^{2 p}(\Omega)}^{2} .
$$

Now, from (2.7) and the continuous embeddings $\mathbf{W}_{0}^{1, p}(\Omega) \subset \mathbf{H}_{0}^{1}(\Omega) \subset \mathbf{L}^{2 p}(\Omega)$ and $\mathbf{W}^{-1, p}(\Omega) \subset \mathbf{H}^{-1}(\Omega)$ for $p>2$, we infer from the above inequality and $(2.7)$

$$
\|\operatorname{div}(\mathbf{y} \otimes \mathbf{y})\|_{\mathbf{W}^{-1, p}(\Omega)} \leq C\|\mathbf{f}\|_{\mathbf{W}^{-1, p}(\Omega)}^{2} \quad \forall p>2 .
$$

Hence, $\mathbf{g} \in \mathbf{W}^{-1, p}(\Omega)$ and

$$
\|\mathbf{g}\|_{\mathbf{W}^{-1, p}(\Omega)} \leq\|\mathbf{f}\|_{\mathbf{W}^{-1, p}(\Omega)}+C\left\|_{\mathbf{f}}\right\|_{\mathbf{W}^{-1, p}(\Omega)}^{2} \quad \forall p>2 .
$$

Combining this inequality with (2.9) we obtain (2.4). Inequality (2.5) follows from (2.9), (2.10), and the fact that $\mathbf{W}_{0}^{1, p}(\Omega) \subset \mathbf{L}^{\frac{2 p}{2-p}}(\Omega) \subset \mathbf{L}^{2 p}(\Omega)$ for $1<p<2$.

Finally, we prove (2.6). We consider the Stokes problem

$$
\left\{\begin{array}{l}
-\nu \Delta \phi+\nabla \pi=\mathbf{f} \text { in } \Omega, \\
\operatorname{div} \phi=0 \text { in } \Omega, \phi=0 \text { on } \Gamma .
\end{array}\right.
$$

Using again [15, Theorem IV.6.1] as above we infer that $\phi \in \mathbf{W}_{0}^{1, p}(\Omega)$ and for some constant depending on $p$ but independent of $\mathbf{f}$

$$
\|\phi\|_{\mathbf{W}_{0}^{1, p}(\Omega)}+\|\pi\|_{L^{p}(\Omega)} \leq C_{1}\|\mathbf{f}\|_{\mathbf{W}^{-1, p}(\Omega)} .
$$

Copyright $@$ by SIAM. Unauthorized reproduction of this article is prohibited. 
Set $(\mathbf{z}, \mathfrak{q})=(\mathbf{y}-\boldsymbol{\phi}, \mathfrak{p}-\pi)$. From the above equation and (2.1) we obtain

$$
\left\{\begin{array}{l}
-\nu \Delta \mathbf{z}+\nabla \mathfrak{q}=-\operatorname{div}(\mathbf{y} \otimes \mathbf{y}) \text { in } \Omega \\
\operatorname{div} \mathbf{z}=0 \text { in } \Omega, \mathbf{z}=0 \text { on } \Gamma .
\end{array}\right.
$$

For every $\mathbf{w} \in \mathbf{H}_{0}^{1}(\Omega)$ we have

$$
-\langle\operatorname{div}(\mathbf{y} \otimes \mathbf{y}), \mathbf{w}\rangle=\int_{\Omega}(\mathbf{y} \cdot \nabla) \mathbf{w} \mathbf{y} d x \leq\|\mathbf{y}\|_{\mathbf{L}^{4}(\Omega)}^{2}\|\mathbf{w}\|_{\mathbf{H}_{0}^{1}(\Omega)} .
$$

Hence, $-\operatorname{div}(\mathbf{y} \otimes \mathbf{y}) \in \mathbf{H}^{-1}(\Omega)$ and

$$
\|\operatorname{div}(\mathbf{y} \otimes \mathbf{y})\|_{\mathbf{H}^{-1}(\Omega)} \leq\|\mathbf{y}\|_{\mathbf{L}^{4}(\Omega)}^{2} .
$$

Therefore, $\mathbf{z} \in \mathbf{H}_{0}^{1}(\Omega)$ holds. Multiplying (2.11) by $\mathbf{z}$, integrating by parts, and using that $\mathbf{y}=\mathbf{z}+\boldsymbol{\phi}$ and the properties of the trilinear form $b$, we infer

$$
\begin{aligned}
& \nu\|\mathbf{z}\|_{\mathbf{H}_{0}^{1}(\Omega)}^{2}=\langle-\operatorname{div}(\mathbf{y} \otimes \mathbf{y}), \mathbf{z}\rangle=b(\mathbf{y}, \mathbf{z}, \mathbf{y})=b(\mathbf{y}, \mathbf{z}, \boldsymbol{\phi}) \leq\|\mathbf{y}\|_{\mathbf{L}^{4}(\Omega)}\|\boldsymbol{\phi}\|_{\mathbf{L}^{4}(\Omega)}\|\mathbf{z}\|_{\mathbf{H}_{0}^{1}(\Omega)} \\
& \quad \leq C_{2}\|\mathbf{y}\|_{\mathbf{L}^{4}(\Omega)}\|\boldsymbol{\phi}\|_{\mathbf{W}_{0}^{1, p}(\Omega)}\|\mathbf{z}\|_{\mathbf{H}_{0}^{1}(\Omega)} \leq C_{1} C_{2}\|\mathbf{f}\|_{\mathbf{W}^{-1, p}(\Omega)}\|\mathbf{y}\|_{\mathbf{L}^{4}(\Omega)}\|\mathbf{z}\|_{\mathbf{H}_{0}^{1}(\Omega)} .
\end{aligned}
$$

Here we have used that $\mathbf{W}_{0}^{1, p}(\Omega) \subset \mathbf{L}^{4}(\Omega)$ because $p \geq 4 / 3$. The above inequality leads to

$$
\|\mathbf{z}\|_{\mathbf{H}_{0}^{1}(\Omega)} \leq \frac{C_{1} C_{2}}{\nu}\|\mathbf{f}\|_{\mathbf{W}^{-1, p}(\Omega)}\|\mathbf{y}\|_{\mathbf{L}^{4}(\Omega)}
$$

and, hence

$$
\|\mathbf{y}\|_{\mathbf{W}_{0}^{1, p}(\Omega)} \leq\|\mathbf{z}\|_{\mathbf{W}_{0}^{1, p}(\Omega)}+\|\boldsymbol{\phi}\|_{\mathbf{W}_{0}^{1, p}(\Omega)} \leq M_{p}\|\mathbf{f}\|_{\mathbf{W}^{-1, p}(\Omega)}\left(1+\|\mathbf{y}\|_{\mathbf{L}^{4}(\Omega)}\right),
$$

which proves the estimate (2.6) for $\mathbf{y}$. The estimate for $\mathfrak{p}$ follows from de Rham's theorem.

Remark 2.4. Let us observe that $\mathbf{W}_{0}^{1, p}(\Omega) \subset \mathbf{L}^{\frac{2 p}{2-p}}(\Omega) \subset \mathbf{L}^{2 p}(\Omega) \forall 1<p<2$. Hence, the right-hand side in (2.5) is finite.

Remark 2.5. Theorem 2.3 proves that for $\mathbf{f}_{0} \in \mathbf{W}^{-1, p}(\Omega)$ with $p \in[4 / 3,2)$ and all $\mathbf{u} \in \mathbf{M}(\omega)$ system (1.1) has a solution $(\mathbf{y}, \mathfrak{p}) \in \mathbf{W}_{0}^{1, p}(\Omega) \times L^{p}(\Omega) / \mathbb{R}$, and the following estimate holds

$$
\|\mathbf{y}\|_{\mathbf{W}_{0}^{1, p}(\Omega)}+\|\mathfrak{p}\|_{L^{p}(\Omega)} \leq M_{p}\left(\left\|\mathbf{f}_{0}\right\|_{\mathbf{W}^{-1, p}(\Omega)}+\|\mathbf{u}\|_{\mathbf{M}(\omega)}\right)\left(1+\|\mathbf{y}\|_{\mathbf{L}^{4}(\Omega)}\right) .
$$

Remark 2.6. In dimension 3, the embedding $\mathbf{M}(\Omega) \subset \mathbf{W}^{-1, p}(\Omega)$ is valid only for $p<3 / 2$. However, the existence of a solution of (2.1) in dimension 3 for $\mathbf{f} \in \mathbf{W}^{-1, p}(\Omega)$ with $1<p \leq 3 / 2$ is an open issue; see [18] or [24]. Thus the three-dimensional case will require a treatment that is different of that used in the present paper.

Definition 2.7. Let $(\overline{\mathbf{y}}, \overline{\mathfrak{p}})$ be a solution of (1.1) associated to some control $\overline{\mathbf{u}} \in \mathbf{M}(\omega)$. We say that $\overline{\mathbf{y}}$ is regular if for every $\mathbf{g} \in \mathbf{H}^{-1}(\Omega)$ the system,

$$
\left\{\begin{array}{l}
-\nu \Delta \mathbf{z}+(\overline{\mathbf{y}} \cdot \nabla) \mathbf{z}+(\mathbf{z} \cdot \nabla) \overline{\mathbf{y}}+\nabla \mathfrak{q}=\mathbf{g} \quad \text { in } \Omega, \\
\operatorname{div} \mathbf{z}=0 \text { in } \Omega, \mathbf{z}=0 \text { on } \Gamma,
\end{array}\right.
$$

has a unique solution $(\mathbf{z}, \mathfrak{q}) \in \mathbf{H}_{0}^{1}(\Omega) \times L^{2}(\Omega) / \mathbb{R}$. 
Observe that the above definition implies that the linear operator

$$
\begin{aligned}
T: \mathbf{V} \times L^{2}(\Omega) / \mathbb{R} & \longrightarrow \mathbf{H}^{-1}(\Omega) \\
(\mathbf{z}, \mathfrak{q}) & \longrightarrow-\nu \Delta \mathbf{z}+(\overline{\mathbf{y}} \cdot \nabla) \mathbf{z}+(\mathbf{z} \cdot \nabla) \overline{\mathbf{y}}+\nabla \mathfrak{q}
\end{aligned}
$$

is an isomorphism.

Remark 2.8. Let us mention that the regularity assumption of $\overline{\mathbf{y}}$ fails only if 0 is an eigenvalue of the operator $T$. We recall that the spectrum of $T$ is formed by an unbounded sequence of eigenvalues with no finite accumulation point. This type of assumption on the linearized Navier-Stokes equations has been considered by some authors in different contexts; see, for instance, [6] and [16, section IV-3.1]. In Remark 3.3 , we will show that for $\nu$ large enough the optimal states for problem $(\mathrm{P})$ are regular.

Assumption. From now on it will be assumed that $\mathbf{f}_{0} \in \mathbf{W}^{-1, p}(\Omega)$ and $p \in(4 / 3,2)$ is fixed.

THEOREM 2.9. Let $\overline{\mathbf{y}}$ be a regular solution of (1.1) associated to some control $\mathbf{u} \in \mathbf{M}(\omega)$. Then, for all $\mathbf{g} \in \mathbf{W}^{-1, p}(\Omega)$ system (2.13) has a unique solution $(\mathbf{z}, \mathfrak{q}) \in$ $\mathbf{W}_{0}^{1, p}(\Omega) \times L^{p}(\Omega) / \mathbb{R}$. Hence,

$$
\begin{aligned}
T_{p}: \mathbf{W}_{p} \times L^{p}(\Omega) / \mathbb{R} & \longrightarrow \mathbf{W}^{-1, p}(\Omega) \\
(\mathbf{z}, \mathfrak{q}) & \longrightarrow-\nu \Delta \mathbf{z}+(\overline{\mathbf{y}} \cdot \nabla) \mathbf{z}+(\mathbf{z} \cdot \nabla) \overline{\mathbf{y}}+\nabla \mathfrak{q}
\end{aligned}
$$

is an isomorphism.

Proof. The proof is based on a duality argument, and it is split in several steps.

Step 1. Adjoint system in $\mathbf{V} \times L^{2}(\Omega) / \mathbb{R}$. Given $\boldsymbol{\psi} \in \mathbf{H}^{-1}(\Omega)$, we consider the system

$$
\left\{\begin{array}{l}
-\nu \Delta \phi-(\overline{\mathbf{y}} \cdot \nabla) \phi+(\nabla \overline{\mathbf{y}})^{T} \phi+\nabla \pi=\boldsymbol{\psi} \text { in } \Omega \\
\operatorname{div} \phi=0 \text { in } \Omega, \phi=0 \text { on } \Gamma .
\end{array}\right.
$$

Associated with the above system, we define the operator

$$
\begin{aligned}
S: \mathbf{V} \times L^{2}(\Omega) / \mathbb{R} & \longrightarrow \mathbf{H}^{-1}(\Omega) \\
(\boldsymbol{\phi}, \pi) & \longrightarrow-\nu \Delta \boldsymbol{\phi}-(\overline{\mathbf{y}} \cdot \nabla) \boldsymbol{\phi}+(\nabla \overline{\mathbf{y}})^{T} \boldsymbol{\phi}+\nabla \pi .
\end{aligned}
$$

We prove that $S$ is an isomorphism. To this end, we first establish the following estimate:

$$
\exists C>0:\|\phi\|_{\mathbf{H}_{0}^{1}(\Omega)}+\|\pi\|_{L^{2}(\Omega)} \leq C\|S(\boldsymbol{\phi}, \pi)\|_{\mathbf{H}^{-1}(\Omega)} \quad \forall(\boldsymbol{\phi}, \pi) \in \mathbf{V} \times L^{2}(\Omega) / \mathbb{R} .
$$

Let us take $\mathbf{g} \in \mathbf{H}^{-1}(\Omega)$ arbitrarily and $(\mathbf{z}, \mathfrak{q}) \in \mathbf{V} \times L^{2}(\Omega) / \mathbb{R}$ such that $T(\mathbf{z}, \mathfrak{q})=\mathbf{g}$. Then, integrating by parts and using that $\operatorname{div} \phi=\operatorname{div} \mathbf{z}=0$ we obtain

$$
\begin{aligned}
& \langle\mathbf{g}, \boldsymbol{\phi}\rangle=\langle T(\mathbf{z}, \mathfrak{q}), \boldsymbol{\phi})\rangle=\langle S(\boldsymbol{\phi}, \pi), \mathbf{z})\rangle \\
& \quad \leq\|S(\boldsymbol{\phi}, \pi)\|_{\mathbf{H}^{-1}(\Omega)}\|\mathbf{z}\|_{\mathbf{H}_{0}^{1}(\Omega)} \leq C_{1}\|S(\boldsymbol{\phi}, \pi)\|_{\mathbf{H}^{-1}(\Omega)}\|\mathbf{g}\|_{\mathbf{H}^{-1}(\Omega)} .
\end{aligned}
$$

The last inequality follows from the fact that $T$ is an isomorphism due to the regularity of $\overline{\mathbf{y}}$. Since $\mathbf{g}$ was arbitrary in $\mathbf{H}^{-1}(\Omega)$, we conclude that

$$
\|\phi\|_{\mathbf{H}_{0}^{1}(\Omega)} \leq C_{1}\|S(\phi, \pi)\|_{\mathbf{H}^{-1}(\Omega)} .
$$

Copyright $@$ by SIAM. Unauthorized reproduction of this article is prohibited. 
Now, from this estimate, (2.16) and arguing as in (2.10) with $p=2$ we conclude that

$$
\begin{aligned}
& \|\nabla \pi\|_{\mathbf{H}^{-1}(\Omega)} \leq\|S(\boldsymbol{\phi}, \pi)\|_{\mathbf{H}^{-1}(\Omega)}+\left\|\nu \Delta \phi+(\overline{\mathbf{y}} \cdot \nabla) \boldsymbol{\phi}-(\nabla \overline{\mathbf{y}})^{T} \boldsymbol{\phi}\right\|_{\mathbf{H}^{-1}(\Omega)} \\
& \quad \leq\|S(\boldsymbol{\phi}, \pi)\|_{\mathbf{H}^{-1}(\Omega)}+\left(\nu+C_{2}\|\overline{\mathbf{y}}\|_{\mathbf{L}^{4}(\Omega)}\right)\|\boldsymbol{\phi}\|_{\mathbf{H}_{0}^{1}(\Omega)} \leq C_{3}\|S(\boldsymbol{\phi}, \pi)\|_{\mathbf{H}^{-1}(\Omega)} .
\end{aligned}
$$

Hence, (2.17) is proved.

From (2.17) we deduce that $S$ is an injective operator with a closed range in $\mathbf{H}^{-1}(\Omega)$. Let us prove that it is surjective. We proceed by contradiction. If it is not surjective, then there exists an element $\mathbf{z} \in \mathbf{H}_{0}^{1}(\Omega)$ with $\mathbf{z} \neq 0$ such that $\langle S(\boldsymbol{\phi}, \pi), \mathbf{z}\rangle=0$ for all $(\phi, \pi) \in \mathbf{V} \times L^{2}(\Omega) / \mathbb{R}$. This means that

$$
\int_{\Omega}\left\{\nu \nabla \boldsymbol{\phi} \cdot \nabla \mathbf{z}-\left[(\overline{\mathbf{y}} \cdot \nabla) \boldsymbol{\phi}-(\nabla \overline{\mathbf{y}})^{T} \boldsymbol{\phi}\right] \mathbf{z}+\nabla \pi \mathbf{z}\right\} d x=0 \quad \forall(\boldsymbol{\phi}, \pi) \in \mathbf{V} \times L^{2}(\Omega) / \mathbb{R} .
$$

Setting $\phi=0$ and $\pi$ arbitrary in $L^{2}(\Omega) / \mathbb{R}$, we infer that $\operatorname{div} \mathbf{z}=0$, hence $\mathbf{z} \in \mathbf{V}$. Now, setting $\pi=0$ and $\phi \in \mathbf{V}$ arbitrary we get after integration by parts

$$
\int_{\Omega}\{\nu \nabla \phi \cdot \nabla \mathbf{z}+[(\overline{\mathbf{y}} \cdot \nabla) \mathbf{z}+(\mathbf{z} \cdot \nabla) \overline{\mathbf{y}}] \boldsymbol{\phi}\} d x=0 \quad \forall \boldsymbol{\phi} \in \mathbf{V} .
$$

Once again, from de Rham's theorem we infer the existence of $\mathfrak{q} \in L^{2}(\Omega) / \mathbb{R}$ such that

$$
-\nu \Delta \mathbf{z}+(\overline{\mathbf{y}} \cdot \nabla) \mathbf{z}+(\mathbf{z} \cdot \nabla) \overline{\mathbf{y}}+\nabla \mathfrak{q}=0 .
$$

This means that $T(\mathbf{z}, \mathfrak{q})=0$, and thus $\mathbf{z}=0$, which contradicts our assumption. Thus $S$ is surjective and (2.17) holds. This implies that $S$ is an isomorphism.

Step 2. Adjoint system in $\mathbf{W}_{p^{\prime}} \times L^{p^{\prime}}(\Omega) / \mathbb{R}$. Associated with the system (2.16), we define the operator

$$
\begin{aligned}
S_{p^{\prime}}: \mathbf{W}_{p^{\prime}} \times L^{p^{\prime}}(\Omega) / \mathbb{R} & \longrightarrow \mathbf{W}^{-1, p^{\prime}}(\Omega) \\
(\boldsymbol{\phi}, \pi) & \longrightarrow-\nu \Delta \phi-(\overline{\mathbf{y}} \cdot \nabla) \boldsymbol{\phi}+(\nabla \overline{\mathbf{y}})^{T} \boldsymbol{\phi}+\nabla \pi .
\end{aligned}
$$

We will prove that $S_{p^{\prime}}$ is an isomorphism. First, we prove surjectivity. Let $\psi \in$ $\mathbf{W}^{-1, p^{\prime}}(\Omega)$ be arbitrary. Since $p<2$, we have that $\mathbf{W}^{-1, p^{\prime}}(\Omega) \subset \mathbf{H}^{-1}(\Omega)$. Therefore, there exists $(\phi, \pi) \in \mathbf{V} \times L^{2}(\Omega) / \mathbb{R}$ such that $S(\boldsymbol{\phi}, \pi)=\boldsymbol{\psi}$. This implies that

$$
\left\{\begin{array}{l}
-\nu \Delta \phi+\nabla \pi=\boldsymbol{\psi}+(\overline{\mathbf{y}} \cdot \nabla) \phi-(\nabla \overline{\mathbf{y}})^{T} \phi \text { in } \Omega, \\
\operatorname{div} \phi=0 \text { in } \Omega, \phi=0 \text { on } \Gamma .
\end{array}\right.
$$

Let us prove that the right-hand side of this equation belongs to $\mathbf{W}^{-1, p^{\prime}}(\Omega)$. Let us take $\mathbf{w} \in \mathbf{W}_{0}^{1, p}(\Omega)$, then with the Hölder inequality for $r=\frac{2 p-2}{2-p}$ and $r^{\prime}=\frac{2 p-2}{3 p-4}$

$$
\begin{aligned}
& \left|\int_{\Omega}(\overline{\mathbf{y}} \cdot \nabla) \phi \mathbf{w} d x\right|=\left|\int_{\Omega}(\overline{\mathbf{y}} \cdot \nabla) \mathbf{w} \phi d x\right| \\
& \quad \leq\|\overline{\mathbf{y}} \cdot \boldsymbol{\phi}\|_{\mathbf{L}^{p^{\prime}}(\Omega)}\|\mathbf{w}\|_{\mathbf{W}_{0}^{1, p}(\Omega)} \leq\|\overline{\mathbf{y}}\|_{\mathbf{L}^{\frac{2 p}{2-p}}(\Omega)}\|\boldsymbol{\phi}\|_{\mathbf{L}^{\frac{2 p}{3^{2}-4}}(\Omega)}\|\mathbf{w}\|_{\mathbf{W}_{0}^{1, p}(\Omega)} .
\end{aligned}
$$

From here we deduce

$$
\begin{aligned}
& \|(\overline{\mathbf{y}} \cdot \nabla) \phi\|_{\mathbf{W}^{-1, p^{\prime}}(\Omega)} \leq\|\overline{\mathbf{y}}\|_{\mathbf{L}^{\frac{2 p}{2-p}}(\Omega)}\|\phi\|_{\mathbf{L}^{\frac{2 p}{3 p-4}}(\Omega)} \\
& \quad \leq C_{1}\|\overline{\mathbf{y}}\|_{\mathbf{W}_{0}^{1, p}(\Omega)}\|\boldsymbol{\phi}\|_{\mathbf{H}_{0}^{1}(\Omega)} \leq C_{2}\|\boldsymbol{\psi}\|_{\mathbf{H}^{-1}(\Omega)} \leq C_{3}\|\boldsymbol{\psi}\|_{\mathbf{W}^{-1, p^{\prime}}(\Omega)}
\end{aligned}
$$

Copyright $@$ by SIAM. Unauthorized reproduction of this article is prohibited. 
The estimate for $\left\|(\nabla \overline{\mathbf{y}})^{T} \boldsymbol{\phi}\right\|_{\mathbf{W}^{-1, p^{\prime}}(\Omega)}$ follows in an analogous way. Now, from estimates for the Stokes system (2.18) we obtain the existence of a constant $C$ such that

$$
\|\phi\|_{\mathbf{W}_{0}^{1, p^{\prime}}(\Omega)}+\|\pi\|_{L^{p^{\prime}}(\Omega) / \mathbb{R}} \leq C\|\boldsymbol{\psi}\|_{\mathbf{W}^{-1, p^{\prime}}(\Omega)} ;
$$

see [15, Theorem IV.6.1]. We have proved the surjectivity of $S_{p^{\prime}}$.

Let us establish the injectivity. Assume that $S_{p^{\prime}}(\phi, \pi)=0$ for some $(\phi, \pi) \in$ $\mathbf{W}_{p^{\prime}} \times L^{p^{\prime}}(\Omega) / \mathbb{R}$. Due to the embeddings $\mathbf{W}_{p^{\prime}} \subset \mathbf{V}$ and $L^{p^{\prime}}(\Omega) / \mathbb{R} \subset L^{2}(\Omega) / \mathbb{R}$, we have that $S(\phi, \pi)=S_{p^{\prime}}(\phi, \pi)=0$. Since $S$ is an isomorphism we infer that $(\phi, \pi)=(0,0)$. Hence, $S_{p^{\prime}}$ is an isomorphism.

Step 3. $T_{p}$ is an isomorphism. Let us consider a sequence $\left\{\mathbf{g}_{k}\right\}_{k=1}^{\infty} \subset \mathbf{H}^{-1}(\Omega)$ such that $\mathbf{g}_{k} \rightarrow \mathbf{g}$ in $\mathbf{W}^{-1, p}(\Omega)$. Due to the regularity of $\overline{\mathbf{y}}$, we know that there exists a unique element $\left(\mathbf{z}_{k}, \mathfrak{q}_{k}\right) \in \mathbf{V} \times L^{2}(\Omega) / \mathbb{R}$ such that $T\left(\mathbf{z}_{k}, \mathfrak{q}_{k}\right)=\mathbf{g}_{k}$. We are going to prove the boundedness of $\left\{\left(\mathbf{z}_{k}, \mathfrak{q}_{k}\right)\right\}_{k=1}^{\infty}$ in $\mathbf{W}_{0}^{1, p}(\Omega) \times L^{p}(\Omega) / \mathbb{R}$ by a duality argument. To this end, given $\boldsymbol{\psi} \in \mathbf{W}^{-1, p^{\prime}}(\Omega)$ arbitrarily we take $(\phi, \pi) \in \mathbf{W}_{p^{\prime}} \times L^{p^{\prime}}(\Omega) / \mathbb{R}$ such that $S_{p^{\prime}}(\phi, \pi)=\boldsymbol{\psi}$. Using that $\operatorname{div} \mathbf{z}_{k}=\operatorname{div} \phi=0$ and integrating by parts we infer

$$
\begin{aligned}
& \left\langle\boldsymbol{\psi}, \mathbf{z}_{k}\right\rangle=\left\langle S_{p^{\prime}}(\boldsymbol{\phi}, \pi), \mathbf{z}_{k}\right\rangle=\left\langle T\left(\mathbf{z}_{k}, \mathfrak{q}_{k}\right), \boldsymbol{\phi}\right\rangle=\left\langle\mathbf{g}_{k}, \boldsymbol{\phi}\right\rangle \\
& \quad \leq\left\|\mathbf{g}_{k}\right\|_{\mathbf{W}^{-1, p}(\Omega)}\|\boldsymbol{\phi}\|_{\mathbf{W}_{0}^{1, p^{\prime}}(\Omega)} \leq C_{1}\|\mathbf{g}\|_{\mathbf{W}^{-1, p}(\Omega)}\|\boldsymbol{\psi}\|_{\mathbf{W}^{-1, p^{\prime}}(\Omega)}
\end{aligned}
$$

This implies that

$$
\left\|\mathbf{z}_{k}\right\|_{\mathbf{W}_{0}^{1, p}(\Omega)} \leq C_{1}\|\mathbf{g}\|_{\mathbf{W}^{-1, p}(\Omega)} \quad \forall k .
$$

Thus, on a subsequence, we have that $\mathbf{z}_{k} \rightarrow \mathbf{z}$ in $\mathbf{W}_{0}^{1, p}(\Omega)$. We have $\operatorname{div} \mathbf{z}=0$ and passing to the limit in the variational equation for $\mathbf{z}_{k}$ we get

$$
\int_{\Omega}\{\nu \nabla \mathbf{z} \cdot \nabla \mathbf{w}+[(\overline{\mathbf{y}} \cdot \nabla) \mathbf{z}+(\mathbf{z} \cdot \nabla) \overline{\mathbf{y}}] \mathbf{w}\} d x=\langle\mathbf{g}, \mathbf{w}\rangle \quad \forall \mathbf{w} \in \mathbf{W}_{p^{\prime}} .
$$

By de Rham's theorem we deduce the existence of $\mathfrak{q} \in L^{p}(\Omega) / \mathbb{R}$ such that $(\mathbf{z}, \mathfrak{q})$ satisfies (2.13). We have proved that $T_{p}$ is surjective.

To prove injectivity, let us assume that $T_{p}(\mathbf{z}, \mathfrak{q})=0$ for some $(\mathbf{z}, \mathfrak{q}) \in \mathbf{W}_{p} \times$ $L^{p}(\Omega) / \mathbb{R}$. Therefore, $\left\langle T_{p}(\mathbf{z}, \mathfrak{q}), \boldsymbol{\phi}\right\rangle=0$ for all $\phi \in \mathbf{W}_{0}^{1, p^{\prime}}(\Omega)$. Hence, since $\operatorname{div} \mathbf{z}=0$, this equality implies after integration by parts that

$$
\int_{\Omega}\left\{\nu \nabla \boldsymbol{\phi} \cdot \nabla \mathbf{z}-\left[(\overline{\mathbf{y}} \cdot \nabla) \boldsymbol{\phi}-(\nabla \overline{\mathbf{y}})^{T} \boldsymbol{\phi}\right] \mathbf{z}+\nabla \pi \mathbf{z}\right\} d x=0 \quad \forall(\boldsymbol{\phi}, \pi) \in \mathbf{W}_{p^{\prime}} \times L^{p^{\prime}}(\Omega) / \mathbb{R} .
$$

This means that $\left\langle S_{p^{\prime}}(\phi, \pi), \mathbf{z}\right\rangle=0$ for every $(\phi, \pi) \in \mathbf{W}_{p^{\prime}} \times L^{p^{\prime}}(\Omega) / \mathbb{R}$. From the surjectivity of $S_{p^{\prime}}$ on $\mathbf{W}^{-1, p^{\prime}}(\Omega)$ we deduce that $\mathbf{z}=0$. Now, from $T_{p}(\mathbf{z}, \mathfrak{q})=0$ we deduce that $\nabla \mathfrak{q}=0$ and, hence, $\mathfrak{q}=0$. Finally, since $T_{p}$ is obviously continuous, we conclude that $T_{p}$ is an isomorphism.

The next theorem addresses the differentiability of the relationship $\mathbf{f} \rightarrow(\mathbf{y}, \mathfrak{p})$ around a regular velocity $\overline{\mathbf{y}}$.

Theorem 2.10. Let $(\overline{\mathbf{y}}, \overline{\mathfrak{p}}, \overline{\mathbf{u}}) \in \mathbf{W}_{p} \times L^{p}(\Omega) / \mathbb{R} \times \mathbf{M}(\omega)$ satisfy (1.1). If $\overline{\mathbf{y}}$ is regular, then there exist open neighborhoods $\mathcal{N}_{p}, \mathcal{Y}_{p}$, and $\mathcal{P}_{p}$ of $\mathbf{f}_{0}+\chi_{\omega} \overline{\mathbf{u}}, \overline{\mathbf{y}}$ and $\overline{\mathfrak{p}}$ in $\mathbf{W}^{-1, p}(\Omega), \mathbf{W}_{p}$ and $L^{p}(\Omega) / \mathbb{R}$, respectively, and a $C^{\infty}$ mapping $\mathcal{G}: \mathcal{N}_{p} \longrightarrow \mathcal{Y}_{p} \times \mathcal{P}_{p}$ 
such that $\mathcal{G}\left(\mathbf{f}_{0}+\chi_{\omega} \overline{\mathbf{u}}\right)=(\overline{\mathbf{y}}, \overline{\mathfrak{p}})$ and $\left(\mathbf{y}_{\mathbf{f}}, \mathfrak{p}_{\mathbf{f}}\right)=\mathcal{G}(\mathbf{f}), f \in \mathcal{N}_{p}$, is the only solution of (2.1) in $\mathcal{Y}_{p} \times \mathcal{P}_{p}$. Moreover, $\mathcal{N}_{p}$ can be taken such that $\mathcal{G}^{\prime}(\mathbf{f}): \mathbf{W}^{-1, p}(\Omega) \longrightarrow \mathbf{W}_{p} \times L^{p}(\Omega) / \mathbb{R}$ is an isomorphism for each $\mathbf{f} \in \mathcal{N}_{p}$. Furthermore, if we denote $\left(\mathbf{z}_{\mathbf{g}}, \mathfrak{q}_{\mathbf{g}}\right)=\mathcal{G}^{\prime}(\mathbf{f}) \mathbf{g}$ and $(\mathbf{z}, \mathfrak{q})=\mathcal{G}^{\prime \prime}(\mathbf{f})\left(\mathbf{g}_{1}, \mathbf{g}_{2}\right)$ with $\mathbf{g}, \mathbf{g}_{i} \in \mathbf{W}^{-1, p}(\Omega)$, then the following relations are fulfilled:

$$
\left\{\begin{array}{l}
-\nu \Delta \mathbf{z}_{\mathbf{g}}+\left(\mathbf{y}_{\mathbf{f}} \cdot \nabla\right) \mathbf{z}_{\mathbf{g}}+\left(\mathbf{z}_{\mathbf{g}} \cdot \nabla\right) \mathbf{y}_{\mathbf{f}}+\nabla \mathfrak{q}_{\mathbf{g}}=\mathbf{g} \text { in } \Omega \\
\operatorname{div} \mathbf{z}_{\mathbf{g}}=0 \text { in } \Omega, \mathbf{z}_{\mathbf{g}}=0 \text { on } \Gamma
\end{array}\right.
$$

and

$$
\left\{\begin{array}{l}
-\nu \Delta \mathbf{z}+\left(\mathbf{y}_{\mathbf{f}} \cdot \nabla\right) \mathbf{z}+(\mathbf{z} \cdot \nabla) \mathbf{y}_{\mathbf{f}}+\nabla \mathfrak{q}=-\left(\mathbf{z}_{\mathbf{g}_{2}} \cdot \nabla\right) \mathbf{z}_{\mathbf{g}_{1}}-\left(\mathbf{z}_{\mathbf{g}_{1}} \cdot \nabla\right) \mathbf{z}_{\mathbf{g}_{2}} \\
\operatorname{div} \mathbf{z}=0 \text { in } \Omega, \mathbf{z}=0 \text { on } \Gamma .
\end{array}\right.
$$

Proof. The proof is based on the implicit function theorem. For this purpose we define

$$
\begin{gathered}
\mathcal{F}: \mathbf{W}_{p} \times L^{p}(\Omega) / \mathbb{R} \times \mathbf{W}^{-1, p}(\Omega) \longrightarrow \mathbf{W}^{-1, p}(\Omega), \\
\mathcal{F}(\mathbf{y}, \mathfrak{p}, \mathbf{f})=-\nu \Delta \mathbf{y}+(\mathbf{y} \cdot \nabla) \mathbf{y}+\nabla \mathfrak{p}-\mathbf{f} .
\end{gathered}
$$

It is easy to check that $\mathcal{F}$ is well defined and it is of class $C^{\infty}$. Moreover, we have $\mathcal{F}(\overline{\mathbf{y}}, \overline{\mathfrak{p}}, \overline{\mathbf{u}})=0$ and

$$
\frac{\partial \mathcal{F}}{\partial(\mathbf{y}, \mathfrak{p})}\left(\overline{\mathbf{y}}, \overline{\mathfrak{p}}, \mathbf{f}_{0}+\chi_{\omega} \overline{\mathbf{u}}\right)(\mathbf{z}, \mathfrak{q})=-\nu \Delta \mathbf{z}+(\overline{\mathbf{y}} \cdot \nabla) \mathbf{z}+(\mathbf{z} \cdot \nabla) \overline{\mathbf{y}}+\nabla \mathfrak{q}=T_{p}(\mathbf{z}, \mathfrak{q}) .
$$

According to Theorem 2.9 this is an isomorphism. Then, the implicit function theorem implies the existence of open neighborhoods $\mathcal{N}_{p}, \mathcal{Y}_{p}$, and $\mathcal{P}_{p}$ of $\mathbf{f}_{0}+\chi_{\omega} \overline{\mathbf{u}}, \overline{\mathbf{y}}$ and $\overline{\mathfrak{p}}$ in $\mathbf{W}^{-1, p}(\Omega), \mathbf{W}_{p}$ and $L^{p}(\Omega) / \mathbb{R}$, respectively, and a $C^{\infty}$ mapping $\mathcal{G}: \mathcal{N}_{p} \longrightarrow$ $\mathcal{Y}_{p} \times \mathcal{P}_{p}$ such that $\mathcal{G}\left(\mathbf{f}_{0}+\chi_{\omega} \overline{\mathbf{u}}\right)=(\overline{\mathbf{y}}, \overline{\mathfrak{p}})$ and $\left(\mathbf{y}_{\mathbf{f}}, \mathfrak{p}_{\mathbf{f}}\right)=\mathcal{G}(\mathbf{f})$ is the only solution of $(2.1)$ in $\mathcal{Y}_{p} \times \mathcal{P}_{p}$. Since $\mathcal{G}^{\prime}: \mathcal{N}_{p} \longrightarrow \mathcal{L}\left(\mathbf{W}^{-1, p}(\Omega), \mathbf{W}_{p} \times L^{p}(\Omega) / \mathbb{R}\right)$ is continuous and $\mathcal{G}^{\prime}\left(\mathbf{f}_{0}+\chi_{\omega} \overline{\mathbf{u}}\right)=T_{p}^{-1}$ is an isomorphism, then $\mathcal{G}^{\prime}(\mathbf{f})$ is also an isomorphism if $\mathbf{f} \in \mathcal{N}_{p}$ and this open neighborhood of $\mathbf{f}_{0}+\chi_{\omega} \overline{\mathbf{u}}$ is small enough. Finally, (2.19) and (2.20) follow differentiating the identity $\mathcal{F}(\mathcal{G}(\mathbf{f}), \mathbf{f})=0$.

Corollary 2.11. Let $(\overline{\mathbf{y}}, \overline{\mathfrak{p}}, \overline{\mathbf{u}})$ satisfy the assumptions of Theorem 2.10. Then there exists an open neighborhood $\mathcal{U}_{p}$ of $\overline{\mathbf{u}}$ in $\mathbf{M}(\omega)$ and a $C^{\infty}$ mapping (with respect to the norm in $\mathbf{M}(\omega)) G: \mathcal{U}_{p} \longrightarrow \mathcal{Y}_{p} \times \mathcal{P}_{p}$ such that $G(\overline{\mathbf{u}})=(\overline{\mathbf{y}}, \overline{\mathfrak{p}})$ and $\left(\mathbf{y}_{u}, \mathfrak{p}_{u}\right)=G(\mathbf{u})$ is the unique solution in $\mathcal{Y}_{p} \times \mathcal{P}_{p}$ of (1.1). Furthermore, the derivatives $G^{\prime}(\mathbf{u}) \mathbf{v}$ and $G^{\prime \prime}(\mathbf{u})\left(\mathbf{v}_{1}, \mathbf{v}_{2}\right)$, for $\mathbf{v}, \mathbf{v}_{i} \in \mathbf{M}(\omega)$, are the solutions of (2.19) and (2.20) replacing $\mathbf{g}$ and $\left(\mathbf{y}_{\mathbf{f}}, \mathfrak{q}_{\mathbf{f}}\right)$ by $\chi_{\omega} \mathbf{v}$ and $\left(\mathbf{y}_{\mathbf{u}}, \mathfrak{q}_{\mathbf{v}}\right)$, respectively.

Proof. Since the mapping $\iota: \mathbf{M}(\omega) \hookrightarrow \mathbf{W}^{-1, p}(\Omega)$ defined by $\iota(\mathbf{u})=\mathbf{f}_{0}+\chi_{\omega} \mathbf{u}$ is continuous and affine, then $\mathcal{U}_{p}=\iota^{-1}\left(\mathcal{N}_{p}\right)$ is an open set in $\mathbf{M}(\omega)$ containing $\overline{\mathbf{u}}$. Now, with $G=\mathcal{G} \circ \iota$ the statements of the corollary follow.

3. Existence of solutions of $(\mathrm{P})$ and first order optimality conditions. In this section, we prove the existence of at least one solution of $(\mathrm{P})$. Then, assuming the regularity of the optimal velocity, we derive the first order optimality conditions and deduce the sparsity structure of the optimal controls.

THEOREM 3.1. There exists at least one solution $(\overline{\mathbf{y}}, \overline{\mathbf{u}})$ of $(\mathrm{P})$. 
Proof. Let $\mathbf{y}_{0}$ be a solution of (1.1) associated to the control $\mathbf{u}=0$. It is obvious that $\left(\mathbf{y}_{0}, 0\right)$ is a feasible point of $(\mathrm{P})$. We assume that $\mathbf{y}_{d} \neq \mathbf{y}_{0}$, otherwise $\left(\mathbf{y}_{0}, 0\right)$ is the unique solution of $(\mathrm{P})$. Take a minimizing sequence $\left\{\left(\mathbf{y}_{k}, \mathbf{u}_{k}\right)\right\}_{k=1}^{\infty}$ with

$$
\mathcal{J}\left(\mathbf{y}_{k}, \mathbf{u}_{k}\right) \leq \mathcal{J}\left(\mathbf{y}_{0}, 0\right)=\frac{1}{2}\left\|\mathbf{y}_{0}-\mathbf{y}_{d}\right\|_{\mathbf{L}^{2}(\Omega)}^{2} \quad \forall k \geq 1 .
$$

This implies that $\left\{\left(\mathbf{y}_{k}, \mathbf{u}_{k}\right)\right\}_{k=1}^{\infty}$ is bounded in $\mathbf{L}^{2}(\Omega) \times \mathbf{M}(\omega)$. Let us prove that $\left\{\mathbf{y}_{k}\right\}_{k=1}^{\infty}$ is bounded in $\mathbf{W}_{0}^{1, p}(\Omega)$. Since $p>\frac{4}{3}$, then the embedding $\mathbf{W}_{0}^{1, p}(\Omega) \subset \mathbf{L}^{4}(\Omega)$ is compact. From (2.12) we obtain

$$
\begin{aligned}
& \left\|\mathbf{y}_{k}\right\|_{\mathbf{W}_{0}^{1, p}(\Omega)}+\left\|\mathfrak{p}_{k}\right\|_{\mathbf{L}^{p}(\Omega)} \leq M_{p}\left(\left\|\mathbf{f}_{0}\right\|_{\mathbf{W}^{-1, p}(\Omega)}+\left\|\mathbf{u}_{k}\right\|_{\mathbf{M}(\omega)}\right)\left(1+\left\|\mathbf{y}_{k}\right\|_{\mathbf{L}^{4}(\Omega)}\right) \\
& \quad \leq M_{p}\left(\left\|\mathbf{f}_{0}\right\|_{\mathbf{W}^{-1, p}(\Omega)}+\frac{1}{2}\left\|\mathbf{y}_{0}-\mathbf{y}_{d}\right\|_{\mathbf{L}^{2}(\Omega)}^{2}\right)\left(1+\left\|\mathbf{y}_{k}\right\|_{\mathbf{L}^{4}(\Omega)}\right) .
\end{aligned}
$$

Applying the Lions lemma $\left[22\right.$, Lemma 2.6.1] to the spaces $\mathbf{W}_{0}^{1, p}(\Omega) \subset \mathbf{L}^{4}(\Omega) \subset \mathbf{L}^{2}(\Omega)$ we deduce the existence of a constant $C>0$ such that

$$
\left\|\mathbf{y}_{k}\right\|_{\mathbf{L}^{4}(\Omega)} \leq \frac{1}{2 M_{p}}\left(\left\|\mathbf{f}_{0}\right\|_{\mathbf{W}^{-1, p}(\Omega)}+\frac{1}{2}\left\|\mathbf{y}_{0}-\mathbf{y}_{d}\right\|_{\mathbf{L}^{2}(\Omega)}^{2}\right)^{-1}\left\|\mathbf{y}_{k}\right\|_{\mathbf{W}_{0}^{1, p}(\Omega)}+C\left\|\mathbf{y}_{k}\right\|_{\mathbf{L}^{2}(\Omega)} .
$$

The last two inequalities imply

$$
\begin{aligned}
& \left\|\mathbf{y}_{k}\right\|_{\mathbf{W}_{0}^{1, p}(\Omega)}+\left\|\mathfrak{p}_{k}\right\|_{\mathbf{L}^{p}(\Omega)} \leq \frac{1}{2}\left\|\mathbf{y}_{k}\right\|_{\mathbf{W}_{0}^{1, p}(\Omega)} \\
& \quad+M_{p}\left(\left\|\mathbf{f}_{0}\right\|_{\mathbf{W}^{-1, p}(\Omega)}+\frac{1}{2}\left\|\mathbf{y}_{0}-\mathbf{y}_{d}\right\|_{\mathbf{L}^{2}(\Omega)}^{2}\right)\left(1+C\left\|\mathbf{y}_{k}\right\|_{\mathbf{L}^{2}(\Omega)}\right)
\end{aligned}
$$

From here and the inequality $\mathcal{J}\left(\mathbf{y}_{k}, \mathbf{u}_{k}\right) \leq \mathcal{J}\left(\mathbf{y}_{0}, 0\right)$ we deduce

$$
\begin{aligned}
& \frac{1}{2}\left\|\mathbf{y}_{k}\right\|_{\mathbf{W}_{0}^{1, p}(\Omega)}+\left\|\mathfrak{p}_{k}\right\|_{\mathbf{L}^{p}(\Omega)} \\
& \quad \leq M_{p}\left(\left\|\mathbf{f}_{0}\right\|_{\mathbf{W}^{-1, p}(\Omega)}+\frac{1}{2}\left\|\mathbf{y}_{0}-\mathbf{y}_{d}\right\|_{\mathbf{L}^{2}(\Omega)}^{2}\right)\left(1+C\left[\left\|\mathbf{y}_{d}\right\|_{\mathbf{L}^{2}(\Omega)}+\left\|\mathbf{y}_{0}-\mathbf{y}_{d}\right\|_{\mathbf{L}^{2}(\Omega)}\right]\right) .
\end{aligned}
$$

Then, we can extract a subsequence, denoted in the same way, such that $\left(\mathbf{y}_{k}, \mathfrak{p}_{k}\right) \rightarrow$ $(\overline{\mathbf{y}}, \overline{\mathfrak{p}})$ in $\mathbf{W}_{0}^{1, p}(\Omega) \times \mathbf{L}^{p}(\Omega)$ and $\mathbf{u}_{k} \stackrel{*}{\rightarrow} \overline{\mathbf{u}}$ in $\mathbf{M}(\omega)$. We prove that $(\overline{\mathbf{y}}, \overline{\mathfrak{p}})$ is a solution of (1.1) corresponding to $\overline{\mathbf{u}}$. To this end, we pass to the limit in the identity

$$
\int_{\Omega}\left(\nabla \mathbf{y}_{k} \nabla \mathbf{z}-\left(\mathbf{y}_{k} \cdot \nabla\right) \mathbf{z} \mathbf{y}_{k}\right) d x-\int_{\Omega} \mathfrak{p}_{k} \operatorname{div} \mathbf{z} d x=\left\langle\mathbf{f}_{0}, \mathbf{z}\right\rangle+\int_{\Omega} \mathbf{z} d \mathbf{u}_{k} \quad \forall \mathbf{z} \in \mathbf{W}_{0}^{1, p^{\prime}}(\Omega) .
$$

The only delicate point in this limit concerns the nonlinear term. Observe that since $\mathbf{W}_{0}^{1, p}(\Omega)$ is compactly embedded in $\mathbf{L}^{2 p}(\Omega)$, we obtain $\mathbf{y}_{k} \rightarrow \overline{\mathbf{y}}$ in $\mathbf{L}^{2 p}(\Omega)$. Hence, taking $\mathbf{z} \in \mathbf{W}_{0}^{1, p^{\prime}}(\Omega)$ we have

$$
\int_{\Omega}\left(\mathbf{y}_{k} \cdot \nabla\right) \mathbf{z y}_{k} d x \rightarrow \int_{\Omega}(\overline{\mathbf{y}} \cdot \nabla) \mathbf{z} \overline{\mathbf{y}} d x .
$$

Therefore, we infer that $(\overline{\mathbf{y}}, \overline{\mathfrak{p}})$ is a solution of (1.1) associated to $\overline{\mathbf{u}}$. Finally, from the lower weak* semicontinuity of the norm in $\mathbf{M}(\omega)$ we conclude that

$$
\mathcal{J}(\overline{\mathbf{y}}, \overline{\mathbf{u}}) \leq \liminf _{k \rightarrow \infty} \mathcal{J}\left(\mathbf{y}_{k}, \mathbf{u}_{k}\right)=\inf (\mathrm{P}) .
$$

Copyright $@$ by SIAM. Unauthorized reproduction of this article is prohibited. 
Remark 3.2. For estimates below (3.1) it is essential that the norm of $y$ on the right-hand side of (2.12) appears linearly rather than of quadratic nature.

Since $(\mathrm{P})$ is not a convex problem, it is convenient to discuss necessary optimality conditions in the context of local solutions. Here, we say that $(\overline{\mathbf{y}}, \overline{\mathbf{u}})$ is a local solution of $(\mathrm{P})$ if there exists a neighborhood $\mathcal{A}$ of $(\overline{\mathbf{y}}, \overline{\mathbf{u}})$ in $\mathbf{W}_{0}^{1, p}(\Omega) \times \mathbf{M}(\omega)$ such that $\mathcal{J}(\overline{\mathbf{y}}, \overline{\mathbf{u}}) \leq \mathcal{J}(\mathbf{y}, \mathbf{u})$ for all $(\mathbf{y}, \mathbf{u}) \in \mathcal{A}$. If the inequality is strict for all $(\mathbf{y}, \mathbf{u}) \in \mathcal{A} \backslash\{(\overline{\mathbf{y}}, \overline{\mathbf{u}})\}$, we say that $(\overline{\mathbf{y}}, \overline{\mathbf{u}})$ is a strict local solution. We will also consider local solutions in the $\mathbf{W}_{0}^{1, p}(\Omega) \times \mathbf{W}^{-1, p}(\Omega)$ topology. Let us observe that the continuous embedding $\mathbf{M}(\omega) \subset \mathbf{W}^{-1, p}(\Omega)$ implies that any local solution in the $\mathbf{W}_{0}^{1, p}(\Omega) \times \mathbf{W}^{-1, p}(\Omega)$ topology is also a local solution in the $\mathbf{W}_{0}^{1, p}(\Omega) \times \mathbf{M}(\omega)$ topology.

In the rest of this section, $(\overline{\mathbf{y}}, \overline{\mathbf{u}})$ will be a local solution such that $\overline{\mathbf{y}}$ is regular. In this case, Corollary 2.11 implies the existence of neighborhoods $\mathcal{U}_{p}, \mathcal{Y}_{p}$ and $\mathcal{P}_{p}$ of $\overline{\mathbf{u}}, \overline{\mathbf{y}}$ and $\overline{\mathfrak{p}}$ in $\mathbf{M}(\omega), \mathbf{W}_{0}^{1, p}(\Omega)$ and $L^{p}(\Omega) / \mathbb{R}$, and a $C^{\infty}$ function $G: \mathcal{U}_{p} \longrightarrow \mathcal{Y}_{p} \times \mathcal{P}_{p}$ such that $G(\overline{\mathbf{u}})=(\overline{\mathbf{y}}, \overline{\mathfrak{p}})$ and $\left(\mathbf{y}_{\mathbf{u}}, \mathfrak{p}_{\mathbf{u}}\right)=G(\mathbf{u})$ is the unique solution of (1.1) in $\mathcal{Y}_{p} \times \mathcal{P}_{p}$ associated to $\mathbf{u}$.

Remark 3.3. Here, we show that $\overline{\mathbf{y}}$ is regular if the kinematic viscosity $\nu$ is big enough. Arguing as in the proof of the previous theorem, we can prove that if $\mathcal{J}(\overline{\mathbf{y}}, \overline{\mathbf{u}}) \leq \mathcal{J}\left(\mathbf{y}_{0}, 0\right)$, then the following estimate holds:

$$
\begin{aligned}
& \|\overline{\mathbf{y}}\|_{\mathbf{W}_{0}^{1, p}(\Omega)} \leq \bar{M}_{p} \\
& \quad=M_{p}\left(2\left\|\mathbf{f}_{0}\right\|_{\mathbf{W}^{-1, p}(\Omega)}+\left\|\mathbf{y}_{0}-\mathbf{y}_{d}\right\|_{\mathbf{L}^{2}(\Omega)}^{2}\right)\left(1+C\left[\left\|\mathbf{y}_{d}\right\|_{\mathbf{L}^{2}(\Omega)}+\left\|\mathbf{y}_{0}-\mathbf{y}_{d}\right\|_{\mathbf{L}^{2}(\Omega)}\right]\right) .
\end{aligned}
$$

Tracking the proof of this estimate it is easy to check that $\bar{M}_{p}=K_{p} / \nu$ for some constant $K_{p}$ independent of $\nu$. Let us study the coercivity of the bilinear form associated to the operator $T$ on the space $\mathbf{V}$ :

$$
\begin{aligned}
& \nu \int_{\Omega}\left[\mid \nabla \mathbf{z} \|^{2}+(\overline{\mathbf{y}} \cdot \nabla) \mathbf{z z}+(\mathbf{z} \cdot \nabla) \overline{\mathbf{y}} \mathbf{z}\right] d x=\nu \int_{\Omega}\left[\mid \nabla \mathbf{z} \|^{2}-(\mathbf{z} \cdot \nabla) \mathbf{z} \overline{\mathbf{y}}\right] d x \\
& \quad \geq \nu\|\nabla \mathbf{z}\|_{\mathbf{L}^{2}(\Omega)}^{2}-\|\overline{\mathbf{y}}\|_{\mathbf{L}^{4}(\Omega)}\|\mathbf{z}\|_{\mathbf{L}^{4}(\Omega)}\|\nabla \mathbf{z}\|_{\mathbf{L}^{2}(\Omega)} \\
& \quad \geq \nu\|\nabla \mathbf{z}\|_{\mathbf{L}^{2}(\Omega)}^{2}-C_{\Omega, p}\|\overline{\mathbf{y}}\|_{\mathbf{W}_{0}^{1, p}(\Omega)}\|\nabla \mathbf{z}\|_{\mathbf{L}^{2}(\Omega)}^{2} \geq\left(\nu-\frac{C_{\Omega, p} K_{p}}{\nu}\right)\|\nabla \mathbf{z}\|_{\mathbf{L}^{2}(\Omega)}^{2} .
\end{aligned}
$$

Then, the bilinear form is coercive if $\nu>\sqrt{C_{\Omega, p} K_{p}}$. Hence, the existence and uniqueness of the velocity $\mathbf{z}$ follows from the Lax-Milgram theorem and the associated pressure is deduced by de Rham's theorem. This proves that $\overline{\mathbf{y}}$ is regular if $\mathcal{J}(\overline{\mathbf{y}}, \overline{\mathbf{u}}) \leq \mathcal{J}\left(\mathbf{y}_{0}, 0\right)$ and $\nu>\sqrt{C_{\Omega, p} K_{p}}$.

For the local analysis of $(\mathrm{P})$, we introduce the functions $F: \mathcal{U}_{p} \longrightarrow \mathbb{R}$ and $j: \mathbf{M}(\omega) \longrightarrow \mathbb{R}$ by

$$
F(u)=\frac{1}{2} \int_{\Omega}\left|\mathbf{y}_{\mathbf{u}}-\mathbf{y}_{d}\right|^{2} d x \quad \text { and } \quad j(\mathbf{u})=\|\mathbf{u}\|_{\mathbf{M}(\omega)} .
$$

Additionally we set $J: \mathcal{U}_{p} \longrightarrow \mathbb{R}$ as $J(\mathbf{u})=\mathcal{J}\left(\mathbf{y}_{\mathbf{u}}, \mathbf{u}\right)=F(\mathbf{u})+\alpha j(\mathbf{u})$. With this notation and the above discussion, we have that $\overline{\mathbf{u}}$ is a local solution in the $\mathbf{M}(\omega)$ topology of the problem

$$
\left(\mathrm{P}_{\mathrm{loc}}\right) \min _{\mathbf{u} \in \mathcal{U}_{p}} J(\mathbf{u}) .
$$

By assuming the regularity of $\overline{\mathbf{y}}$ we have reduced the problem $(\mathrm{P})$ with two variables and a PDE constraint to an unconstrained problem in one variable. In addition the functional $F$ involved in $J$ is differentiable as we prove next. 
THEOREM 3.4. The functional $F$ is of class $C^{\infty}$ and the following identities hold

$$
\begin{aligned}
& F^{\prime}(\mathbf{u}) \mathbf{v}=\int_{\Omega} \boldsymbol{\varphi}_{\mathbf{u}}(x) d \mathbf{v}(x), \\
& F^{\prime \prime}(\mathbf{u}) \mathbf{v}^{2}=\int_{\Omega}\left\{\left|\mathbf{z}_{\mathbf{v}}\right|^{2}-2\left(\mathbf{z}_{\mathbf{v}} \cdot \nabla\right) \mathbf{z}_{\mathbf{v}} \varphi_{\mathbf{u}}\right\} d x
\end{aligned}
$$

for all $\mathbf{v} \in \mathbf{M}(\omega)$, where $\mathbf{z}_{\mathbf{v}}=G^{\prime}(\mathbf{u}) \mathbf{v}$ and $\varphi_{\mathbf{u}} \in \mathbf{W}_{0}^{1, p^{\prime}}(\Omega)$ is the adjoint state, the unique solution along with $\pi_{\mathbf{u}}$ of

$$
\left\{\begin{array}{l}
-\nu \Delta \boldsymbol{\varphi}_{\mathbf{u}}-\left(\mathbf{y}_{\mathbf{u}} \cdot \nabla\right) \boldsymbol{\varphi}_{\mathbf{u}}+\left(\nabla \mathbf{y}_{\mathbf{u}}\right)^{T} \boldsymbol{\varphi}_{\mathbf{u}}+\nabla \pi_{\mathbf{u}}=\mathbf{y}_{\mathbf{u}}-\mathbf{y}_{d} \text { in } \Omega \\
\operatorname{div} \boldsymbol{\varphi}_{\mathbf{u}}=0 \text { in } \Omega, \boldsymbol{\varphi}_{\mathbf{u}}=0 \text { on } \Gamma
\end{array}\right.
$$

Proof. The $C^{\infty}$ differentiability of $F$ is a straightforward consequence of Corollary 2.11 and the chain rule. The existence, uniqueness, and regularity of the adjoint state is obtained as follows. First, we recall that $\mathbf{y}-\mathbf{y}_{d} \in \mathbf{L}^{2}(\Omega) \subset \mathbf{W}^{-1, p^{\prime}}(\Omega)$. As established in the proof of Theorem 2.9, (2.16) has a unique solution $(\phi, \pi) \in$ $\mathbf{W}_{0}^{1, p^{\prime}}(\Omega) \times L^{p^{\prime}}(\Omega) / \mathbb{R}$ for every $\boldsymbol{\psi} \in \mathbf{W}^{-1, p^{\prime}}(\Omega)$. Setting $\boldsymbol{\psi}=\mathbf{y}_{\mathbf{u}}-\mathbf{y}_{d}$, we infer that (3.4) has a unique solution $\left(\boldsymbol{\varphi}_{\mathbf{u}}, \pi_{\mathbf{u}}\right) \in \mathbf{W}_{0}^{1, p^{\prime}}(\Omega) \times L^{p^{\prime}}(\Omega) / \mathbb{R}$, and with (2.19)

$$
\left\|\boldsymbol{\varphi}_{\mathbf{u}}\right\|_{\mathbf{W}_{0}^{1, p^{\prime}(\Omega)}}+\left\|\pi_{\mathbf{u}}\right\|_{L^{p^{\prime}}(\Omega) / \mathbb{R}} \leq C\left\|\mathbf{y}_{\mathbf{u}}-\mathbf{y}_{d}\right\|_{\mathbf{L}^{2}(\Omega)} .
$$

Finally, (3.2) and (3.3) follow from (2.20), (2.21), (3.4), and an integration by parts. Recall that $\mathbf{W}_{0}^{1, p^{\prime}}(\Omega) \subset \mathbf{C}_{0}(\omega)$ because $p^{\prime}>2$; hence the integral in (3.2) is well defined.

Contrary to $F$, the functional $j$ is not differentiable, but it is convex and Lipschitz. Hence the directional derivatives $j^{\prime}(\mathbf{u} ; \mathbf{v})$ at every point $\mathbf{u} \in \mathbf{M}(\omega)$ and in every direction $\mathbf{v} \in \mathbf{M}(\omega)$ exist. The functional $j$ is also subdifferentiable at every point $\mathbf{u} \in \mathbf{M}(\omega)$. We recall that $\boldsymbol{\lambda} \in \partial j(\mathbf{u}) \subset \mathbf{M}(\omega)^{*}$ if

$$
\langle\boldsymbol{\lambda}, \mathbf{v}-\mathbf{u}\rangle_{\mathbf{M}(\omega)^{*}, \mathbf{M}(\omega)}+j(\mathbf{u}) \leq j(\mathbf{v}) \quad \forall \mathbf{v} \in \mathbf{M}(\omega) .
$$

This definition is equivalent to the following two relationships:

$$
\begin{aligned}
& \langle\boldsymbol{\lambda}, \mathbf{u}\rangle_{\mathbf{M}(\omega)^{*}, \mathbf{M}(\omega)}=j(\mathbf{u}), \\
& \langle\boldsymbol{\lambda}, \mathbf{v}\rangle_{\mathbf{M}(\omega)^{*}, \mathbf{M}(\omega)} \leq j(\mathbf{v}) \quad \forall \mathbf{v} \in \mathbf{M}(\omega)
\end{aligned}
$$

Indeed, (3.7) is deduced from (3.6) taking $\mathbf{v}=0$ and then $\mathbf{v}=2 \mathbf{u}$, and using that $j(\rho \mathbf{u})=\rho j(\mathbf{u})$ for every $\rho \geq 0$. Inequality (3.8) is an immediate consequence of (3.6) and (3.7). The converse implication is obvious. Next, we derive some important relations between $\boldsymbol{\lambda}$ and $\mathbf{u}$ when $\boldsymbol{\lambda} \in \mathbf{C}_{0}(\omega) \cap \partial j(\mathbf{u})$. To this end, we will distinguish three cases according to the norm ||$_{\mathbb{R}^{2}}$ considered in the definition of \|\|$_{\mathbf{M}(\omega)}$; see (1.2). Let us introduce the following notation for $\mathbf{u}=\left(u_{1}, u_{2}\right)$

$$
\begin{aligned}
& \|\mathbf{u}\|_{1}=\left|\left(\left\|u_{1}\right\|_{M(\omega)},\left\|u_{2}\right\|_{M(\omega)}\right)\right|_{1}=\left\|u_{1}\right\|_{M(\omega)}+\left\|u_{2}\right\|_{M(\omega)}, \\
& \|\mathbf{u}\|_{2}=\left|\left(\left\|u_{1}\right\|_{M(\omega)},\left\|u_{2}\right\|_{M(\omega)}\right)\right|_{2}=\left(\left\|u_{1}\right\|_{M(\omega)}^{2}+\left\|u_{2}\right\|_{M(\omega)}^{2}\right)^{1 / 2}, \\
& \|\mathbf{u}\|_{\infty}=\left|\left(\left\|u_{1}\right\|_{M(\omega)},\left\|u_{2}\right\|_{M(\omega)}\right)\right|_{\infty}=\max \left\{\left\|u_{1}\right\|_{M(\omega)},\left\|u_{2}\right\|_{M(\omega)}\right\} .
\end{aligned}
$$

Copyright $@$ ㅇ by SIAM. Unauthorized reproduction of this article is prohibited. 
We also set $j_{1}(\mathbf{u})=\|\mathbf{u}\|_{1}, j_{2}(\mathbf{u})=\|\mathbf{u}\|_{2}$ and $j_{\infty}(\mathbf{u})=\|\mathbf{u}\|_{\infty}$. Analogously, for any function $\boldsymbol{\lambda}=\left(\lambda_{1}, \lambda_{2}\right) \in \mathbf{C}_{0}(\omega)$ we consider the dual norms

$$
\begin{aligned}
& \|\boldsymbol{\lambda}\|_{\infty}=\left|\left(\left\|\lambda_{1}\right\|_{\infty},\left\|\lambda_{2}\right\|_{\infty}\right)\right|_{\infty}=\max \left\{\left\|\lambda_{1}\right\|_{\infty},\left\|\lambda_{2}\right\|_{\infty}\right\} \\
& \|\boldsymbol{\lambda}\|_{2}=\left|\left(\left\|\lambda_{1}\right\|_{\infty},\left\|\lambda_{2}\right\|_{\infty}\right)\right|_{2}=\left(\left\|\lambda_{1}\right\|_{\infty}^{2}+\left\|\lambda_{2}\right\|_{\infty}^{2}\right)^{1 / 2} \\
& \|\boldsymbol{\lambda}\|_{1}=\left|\left(\left\|\lambda_{1}\right\|_{\infty},\left\|\lambda_{2}\right\|_{\infty}\right)\right|_{1}=\left\|\lambda_{1}\right\|_{\infty}+\left\|\lambda_{2}\right\|_{\infty} .
\end{aligned}
$$

As usual, given a measure $u \in M(\omega)$, we denote the Jordan decomposition of $u$ by $u=u^{+}-u^{-}$.

With the above notations, we have the following result.

Proposition 3.5. Let $\mathbf{u} \in \mathbf{M}(\omega)$ with $\mathbf{u}=\left(u_{1}, u_{2}\right) \neq 0$ and $\boldsymbol{\lambda} \in \mathbf{C}_{0}(\omega) \cap \partial j(\mathbf{u})$. Then, the following properties are fulfilled.

1. If $j=j_{1}$, then $\|\boldsymbol{\lambda}\|_{\infty}=1$. Moreover, if $u_{i} \neq 0$, then $\left\|\lambda_{i}\right\|_{\infty}=1$ and

$$
\left\{\begin{array}{l}
\operatorname{Supp}\left(u_{i}^{+}\right) \subset\left\{x \in \omega: \lambda_{i}(x)=+1\right\} \\
\operatorname{Supp}\left(u_{i}^{-}\right) \subset\left\{x \in \omega: \lambda_{i}(x)=-1\right\}
\end{array}\right.
$$

2. If $j=j_{2}$, then $\|\boldsymbol{\lambda}\|_{2}=1$. Moreover, for $i=1,2$, if $u_{i} \neq 0$, then

$$
\left\{\begin{array}{l}
\operatorname{Supp}\left(u_{i}^{+}\right) \subset\left\{x \in \omega: \lambda_{i}(x)=+\left\|\lambda_{i}\right\|_{\infty}\right\}, \\
\operatorname{Supp}\left(u_{i}^{-}\right) \subset\left\{x \in \omega: \lambda_{i}(x)=-\left\|\lambda_{i}\right\|_{\infty}\right\} .
\end{array}\right.
$$

3. If $j=j_{\infty}$, then $\|\boldsymbol{\lambda}\|_{1}=1$. Moreover, for $i=1,2$, if $u_{i} \neq 0$, then (3.16) holds.

Proof. Case $j=j_{1}$. Taking $\mathbf{v}=\left(v_{1}, u_{2}\right)$ in (3.6), we get

$$
\int_{\omega} \lambda_{1}(x) d\left(v_{1}-u_{1}\right)(x)+\left\|u_{1}\right\|_{M(\omega)} \leq\left\|v_{1}\right\|_{M(\omega)} \quad \forall v_{1} \in M(\omega) .
$$

Analogously to (3.7)-(3.8), the above inequality is equivalent to

$$
\begin{aligned}
& \int_{\omega} \lambda_{1}(x) d u_{1}(x)=\left\|u_{1}\right\|_{M(\omega)}, \\
& \int_{\omega} \lambda_{1}(x) d v_{1}(x) \leq\left\|v_{1}\right\|_{M(\omega)} \quad \forall v_{1} \in M(\omega) .
\end{aligned}
$$

From the second inequality, it follows that $\left\|\lambda_{1}\right\|_{\infty} \leq 1$. Moreover, if $u_{1} \neq 0$, the first inequality implies that $\left\|\lambda_{1}\right\|_{\infty}=1$. The relations (3.15), for $i=1$, were proved in [3, Lemma 3.4]. In the same way we can argue for $i=2$. Finally, since $\mathbf{u} \neq 0$, then the identity $\left\|\lambda_{i}\right\|_{\infty}=1$ holds for at least one $i$. Therefore, we have $\|\boldsymbol{\lambda}\|_{\infty}=1$.

Case $j=j_{2}$. From (3.7)-(3.8) we infer

$$
\begin{aligned}
& \int_{\omega} \lambda_{1}(x) d u_{1}(x)+\int_{\omega} \lambda_{2}(x) d u_{2}(x)=\|\mathbf{u}\|_{2}, \\
& \int_{\omega} \lambda_{1}(x) d v_{1}(x)+\int_{\omega} \lambda_{2}(x) d v_{2}(x) \leq\|\mathbf{v}\|_{2} \quad \forall \mathbf{v} \in \mathbf{M}(\omega) .
\end{aligned}
$$

Copyright $@$ by SIAM. Unauthorized reproduction of this article is prohibited. 
Using (3.17) we obtain

$$
\begin{aligned}
\|\mathbf{u}\|_{2} & =\int_{\omega} \lambda_{1}(x) d u_{1}(x)+\int_{\omega} \lambda_{2}(x) d u_{2}(x) \\
& \leq \int_{\omega}\left|\lambda_{1}(x)\right| d\left|u_{1}\right|(x)+\int_{\omega}\left|\lambda_{2}(x)\right| d\left|u_{2}\right|(x) \leq\left\|\lambda_{1}\right\|_{\infty}\left\|u_{1}\right\|_{M(\omega)}+\left\|\lambda_{2}\right\|_{\infty}\left\|u_{2}\right\|_{M(\omega)} \\
& \leq\left(\left\|\lambda_{1}\right\|_{\infty}^{2}+\left\|\lambda_{2}\right\|_{\infty}^{2}\right)^{1 / 2}\left(\left\|u_{1}\right\|_{M(\omega)}^{2}+\left\|u_{2}\right\|_{M(\omega)}^{2}\right)^{1 / 2}=\|\boldsymbol{\lambda}\|_{2}\|\mathbf{u}\|_{2} .
\end{aligned}
$$

Since $\mathbf{u} \neq 0$, the above inequality implies that $\|\boldsymbol{\lambda}\|_{2} \geq 1$. To prove the equality we select two points $x_{i} \in \omega, i=1,2$, such that $\left|\lambda_{i}\left(x_{i}\right)\right|=\left\|\lambda_{i}\right\|_{\infty}$. Setting $\mathbf{v}=\left(\lambda_{1}\left(x_{1}\right) \delta_{x_{1}}\right.$, $\left.\lambda_{2}\left(x_{2}\right) \delta_{x_{2}}\right)$ in $(3.18)$ we obtain

$$
\|\boldsymbol{\lambda}\|_{2}^{2}=\int_{\omega} \lambda_{1}(x) d v_{1}(x)+\int_{\omega} \lambda_{2}(x) d v_{2}(x) \leq\|\mathbf{v}\|_{2}=\|\boldsymbol{\lambda}\|_{2} .
$$

Hence, $\|\boldsymbol{\lambda}\|_{2} \leq 1$ and the equality $\|\boldsymbol{\lambda}\|_{2}=1$ holds. Now, we assume that $u_{1} \neq 0$ and denote

$$
\omega_{1}=\left\{x \in \omega:\left|\lambda_{1}(x)\right|=\left\|\lambda_{1}\right\|_{\infty}\right\}
$$

Let $K \subset \omega \backslash \omega_{1}$ an arbitrary compact set. By definition of $\omega_{1}$, we have that $\rho_{K}=$ $\left\|\lambda_{1}\right\|_{C(K)}<\left\|\lambda_{1}\right\|_{\infty}$. For every element $z \in C(K)$ with $\|z\|_{C(K)} \leq\left\|\lambda_{1}\right\|_{\infty}-\rho_{K}$, extended by zero to $\omega$, we have $\left\|\lambda_{1}+z\right\|_{\infty}=\left\|\lambda_{1}\right\|_{\infty}$. Then, using (3.17) we infer

$$
\begin{aligned}
& \int_{\omega}\left(\lambda_{1}(x)+z(x)\right) d u_{1}(x)+\int_{\omega} \lambda_{2}(x) d u_{2}(x) \leq\left(\left\|\lambda_{1}+z\right\|_{\infty}^{2}+\left\|\lambda_{2}\right\|_{\infty}^{2}\right)^{1 / 2}\|\mathbf{u}\|_{2} \\
& =\|\boldsymbol{\lambda}\|_{2}\|\mathbf{u}\|_{2}=\|\mathbf{u}\|_{2}=\int_{\omega} \lambda_{1}(x) d u_{1}(x)+\int_{\omega} \lambda_{2}(x) d u_{2}(x) .
\end{aligned}
$$

This yields

$$
\int_{K} z(x) d u_{1}(x)=\int_{\omega} z(x) d u_{1}(x) \leq 0 \quad \forall z \in C(K):\|z\|_{C(K)} \leq\left\|\lambda_{1}\right\|_{\infty}-\rho_{K} .
$$

This implies that $\left|u_{1}\right|(K)=0$. Since $K$ is an arbitrary compact subset of $\omega \backslash \omega_{1}$ and $u_{1}$ is a regular Borel measure, we conclude that $\left|u_{1}\right|\left(\omega \backslash \omega_{1}\right)=0$. Therefore, the support of $u_{1}$ is contained in $\omega_{1}$. To deduce (3.16), we observe that (3.19) and the fact that $\|\boldsymbol{\lambda}\|_{2}=1$ imply

$$
\int_{\omega} \lambda_{1}(x) d u_{1}(x)+\int_{\omega} \lambda_{2}(x) d u_{2}(x)=\int_{\omega}\left|\lambda_{1}(x)\right| d\left|u_{1}\right|(x)+\int_{\omega}\left|\lambda_{2}(x)\right| d\left|u_{2}\right|(x) .
$$

This identity and the inequalities

$$
\int_{\omega} \lambda_{i}(x) d u_{i}(x) \leq \int_{\omega}\left|\lambda_{i}(x)\right| d\left|u_{i}\right|(x) \text { for } i=1,2,
$$

lead to the identities

$$
\int_{\omega} \lambda_{i}(x) d u_{i}(x)=\int_{\omega}\left|\lambda_{i}(x)\right| d\left|u_{i}\right|(x) \text { for } i=1,2 .
$$

This identity for $i=1$ and the fact that $\operatorname{Supp}\left(u_{1}\right) \subset \omega_{1}$ prove (3.16) for $i=1$. The same arguments can be applied for $i=2$ if $u_{2} \neq 0$.

Copyright (c) by SIAM. Unauthorized reproduction of this article is prohibited. 
Case $j=j_{3}$. The same arguments used in the previous case can be repeated to deal with this case. Let us point out some small differences. The relations (3.19) change this time as follows:

$$
\begin{aligned}
& \|\mathbf{u}\|_{\infty}=\int_{\omega} \lambda_{1}(x) d u_{1}(x)+\int_{\omega} \lambda_{2}(x) d u_{2}(x) \\
& \quad \leq \int_{\omega}\left|\lambda_{1}(x)\right| d\left|u_{1}\right|(x)+\int_{\omega}\left|\lambda_{2}(x)\right| d\left|u_{2}\right|(x) \leq\left\|\lambda_{1}\right\|_{\infty}\left\|u_{1}\right\|_{M(\omega)}+\left\|\lambda_{2}\right\|_{\infty}\left\|u_{2}\right\|_{M(\omega)} \\
& \quad \leq\left(\left\|\lambda_{1}\right\|_{\infty}+\left\|\lambda_{2}\right\|_{\infty}\right) \max \left\{\left\|u_{1}\right\|_{M(\omega)},\left\|u_{2}\right\|_{M(\omega)}\right\}=\|\boldsymbol{\lambda}\|_{1}\|\mathbf{u}\|_{\infty} .
\end{aligned}
$$

This proves that $\|\boldsymbol{\lambda}\|_{1} \geq 1$. To prove the contrary inequality we select two points $x_{1}, x_{2} \in \omega$ such that $\left|\lambda_{i}\left(x_{i}\right)\right|=\left\|\lambda_{i}\right\|_{\infty}$ and set $\mathbf{v}=\left(\operatorname{sign}\left(\lambda_{1}\left(x_{1}\right)\right) \delta_{x_{1}}, \operatorname{sign}\left(\lambda_{2}\left(x_{2}\right)\right) \delta_{x_{2}}\right)$. Thus we obtain with (3.8)

$$
\|\boldsymbol{\lambda}\|_{1}=\int_{\omega} \lambda_{1}(x) d v_{1}(x)+\int_{\omega} \lambda_{2}(x) d v_{2}(x) \leq\|\mathbf{v}\|_{\infty}=1 .
$$

The rest of the proof follows the steps of the second case.

Next, we study the derivatives $j^{\prime}(\mathbf{u} ; \mathbf{v})$ for the previous three cases. To this end, let us introduce some additional notation. Given two measures $u, v \in M(\omega)$, we consider the Lebesgue decomposition of $v=v_{a}+v_{s}$ with respect to $|u|$, where $v_{a}$ is the absolutely continuous part of $v$ with respect to $|u|$, and $v_{s}$ is the singular part. Now, we take the Radon-Nikodym derivative of $v_{a}$ with respect to $|u|, d v_{a}=g_{v} d|u|$. Then we have

$$
\|v\|_{M(\omega)}=\left\|v_{a}\right\|_{M(\omega)}+\left\|v_{s}\right\|_{M(\omega)}=\int_{\omega}\left|g_{v}\right| d|u|+\left\|v_{s}\right\|_{M(\omega)}
$$

In particular, it is obvious that $u$ is absolutely continuous with respect to $|u|$. Hence, we can express $d u=h d|u|$, where $h$ is measurable with respect to $|u|$ and $|h(x)|=1$ for all $x \in \omega, d u^{+}=h^{+} d|u|$ and $d u^{-}=h^{-} d|u|$, where $u=u^{+}-u^{-}$is the Jordan decomposition of $u$. See, for instance, [23, Chapter 6] for details.

Let us define $j_{0}: M(\omega) \longrightarrow[0, \infty)$ by $j_{0}(u)=\|u\|_{M(\omega)}$. The authors proved in $[4$, Proposition 3.3] that

$$
j_{0}^{\prime}(u ; v)=\int_{\omega} g_{v}(x) d u(x)+\left\|v_{s}\right\|_{M(\omega)} \quad \forall u, v \in M(\omega)
$$

where $v=g_{v} d|u|+v_{s}$ is the Lebesgue decomposition of $v$ with respect to $|u|$. Using this identity we get the following result.

Proposition 3.6. For every $\mathbf{u}, \mathbf{v} \in \mathbf{M}(\omega)$ the following identities hold

$$
\begin{aligned}
& j_{1}^{\prime}(\mathbf{u} ; \mathbf{v})=j_{0}^{\prime}\left(u_{1} ; v_{1}\right)+j_{0}^{\prime}\left(u_{2} ; v_{2}\right), \quad \text { if } \mathbf{u}=0 \\
& j_{2}^{\prime}(\mathbf{u} ; \mathbf{v})=\left\{\begin{array}{cl}
1 \\
\frac{1}{\|\mathbf{u}\|_{2}}\left(\left\|u_{1}\right\|_{M(\omega)} j_{0}^{\prime}\left(u_{1} ; v_{1}\right)+\left\|u_{2}\right\|_{M(\omega)} j_{0}^{\prime}\left(u_{2} ; v_{2}\right)\right) & \text { if } \mathbf{u} \neq 0,
\end{array}\right. \\
& j_{\infty}^{\prime}(\mathbf{u} ; \mathbf{v})=\left\{\begin{array}{cl}
j_{0}^{\prime}\left(u_{1} ; v_{1}\right) & \text { if }\left\|u_{1}\right\|_{M(\omega)}>\left\|u_{2}\right\|_{M(\omega)}, \\
j_{0}^{\prime}\left(u_{2} ; v_{2}\right) & \text { if }\left\|u_{1}\right\|_{M(\omega)}<\left\|u_{2}\right\|_{M(\omega)}, \\
\max \left\{j_{0}^{\prime}\left(u_{1} ; v_{1}\right), j_{0}^{\prime}\left(u_{2} ; v_{2}\right)\right\} & \text { if }\left\|u_{1}\right\|_{M(\omega)}=\left\|u_{2}\right\|_{M(\omega)} .
\end{array}\right.
\end{aligned}
$$

Copyright (c) by SIAM. Unauthorized reproduction of this article is prohibited. 
Proof. The identity (3.21) is an immediate consequence of (3.20) and the fact that $j_{1}(\mathbf{u})=j_{0}\left(u_{1}\right)+j_{0}\left(u_{2}\right)$. If $\mathbf{u}=0$, the first identity of $(3.22)$ is obvious. For $\mathbf{u} \neq 0$, the identity is easily deduced from the fact that $j_{2}$ is the composition of the mapping $\mathbf{u} \in \mathbf{M}(\omega) \longrightarrow\left(j_{0}\left(u_{1}\right), j_{0}\left(u_{2}\right)\right) \in \mathbb{R}^{2}$ and the Euclidean norm in $\mathbb{R}^{2}$. The first identity of (3.23) is a straightforward consequence of the fact that $j_{\infty}(\mathbf{u})=j_{0}\left(u_{1}\right)$ and $j_{\infty}(\mathbf{u}+\rho \mathbf{v})=j_{0}\left(u_{1}+\rho v_{1}\right)$ for all $\rho$ sufficiently small if $\left\|u_{1}\right\|_{M(\omega)}>\left\|u_{2}\right\|_{M(\omega)}$. The same argument proves the second identity. To prove the last identity we use that $j_{0}\left(u_{1}\right)=j_{0}\left(u_{2}\right)$ as follows

$$
\begin{aligned}
\lim _{\rho \searrow 0} & \frac{j_{\infty}(\mathbf{u}+\rho \mathbf{v})-j_{\infty}(\mathbf{u})}{\rho}=\lim _{\rho \searrow 0} \frac{\max \left\{j_{0}\left(u_{1}+\rho v_{1}\right), j_{0}\left(u_{2}+\rho v_{2}\right)\right\}-j_{0}\left(u_{1}\right)}{\rho} \\
\quad & =\lim _{\rho \searrow 0} \max \left\{\frac{j_{0}\left(u_{1}+\rho v_{1}\right)-j_{0}\left(u_{1}\right)}{\rho}, \frac{j_{0}\left(u_{2}+\rho v_{2}\right)-j_{0}\left(u_{2}\right)}{\rho}\right\} \\
& =\max \left\{j_{0}^{\prime}\left(u_{1} ; v_{1}\right), j_{0}^{\prime}\left(u_{2} ; v_{2}\right)\right\} .
\end{aligned}
$$

Now, we write the first order optimality conditions satisfied by the local solution $(\overline{\mathbf{y}}, \overline{\mathbf{u}})$.

TheOREm 3.7. Let $(\overline{\mathbf{y}}, \overline{\mathbf{u}})$ be a local solution of $(\mathrm{P})$ such that $\overline{\mathbf{y}}$ is regular. Then, there exists a unique element $(\overline{\boldsymbol{\varphi}}, \bar{\pi}) \in \mathbf{W}_{0}^{1, p^{\prime}}(\Omega) \times L^{p^{\prime}}(\Omega) / \mathbb{R}$ such that

$$
\begin{aligned}
& \left\{\begin{array}{l}
-\nu \Delta \overline{\boldsymbol{\varphi}}-(\overline{\mathbf{y}} \cdot \nabla) \overline{\boldsymbol{\varphi}}+(\nabla \overline{\mathbf{y}})^{T} \overline{\boldsymbol{\varphi}}+\nabla \bar{\pi}=\overline{\mathbf{y}}-\mathbf{y}_{d} \text { in } \Omega, \\
\operatorname{div} \overline{\boldsymbol{\varphi}}=0 \quad \text { in } \Omega, \overline{\boldsymbol{\varphi}}=0 \quad \text { on } \Gamma,
\end{array}\right. \\
& -\frac{1}{\alpha} \overline{\boldsymbol{\varphi}} \in \partial j(\overline{\mathbf{u}}) \cap \mathbf{C}_{0}(\omega) .
\end{aligned}
$$

Proof. First, we recall that $\overline{\mathbf{u}}$ is a local solution of $\left(\mathrm{P}_{\text {loc }}\right)$. We also have that Theorem 3.4 implies that (3.24) has a unique solution $(\bar{\varphi}, \bar{\pi}) \in \mathbf{W}_{0}^{1, p^{\prime}}(\Omega) \times L^{p^{\prime}}(\Omega) / \mathbb{R}$, and

$$
F^{\prime}(\overline{\mathbf{u}}) \mathbf{v}=\int_{\omega} \bar{\varphi}(x) d \mathbf{v}(x) \quad \forall \mathbf{v} \in \mathbf{M}(\omega)
$$

Now, using the convexity of $j$ and the local optimality of $\overline{\mathbf{u}}$ we infer

$$
\begin{aligned}
0 & \leq \lim _{\rho \searrow 0} \frac{J(\overline{\mathbf{u}}+\rho(\mathbf{v}-\overline{\mathbf{u}}))-J(\overline{\mathbf{u}})}{\rho} \leq \lim _{\rho \searrow 0} \frac{F(\overline{\mathbf{u}}+\rho(\mathbf{v}-\overline{\mathbf{u}}))-F(\overline{\mathbf{u}})}{\rho}+\alpha j(\mathbf{v})-\alpha j(\overline{\mathbf{u}}) \\
& =F^{\prime}(\overline{\mathbf{u}})(\mathbf{v}-\overline{\mathbf{u}})+\alpha j(\mathbf{v})-\alpha j(\overline{\mathbf{u}})=\int_{\omega} \bar{\varphi} d(\mathbf{v}-\overline{\mathbf{u}})+\alpha[j(\mathbf{v})-j(\overline{\mathbf{u}})] \forall \mathbf{v} \in \mathbf{M}(\omega) .
\end{aligned}
$$

By definition of $\partial j(\overline{\mathbf{u}})$, the above inequality implies that $-\frac{1}{\alpha} \overline{\boldsymbol{\varphi}} \in \partial j(\overline{\mathbf{u}})$. Recall that the inclusion $\bar{\varphi} \in \mathbf{C}_{0}(\omega)$ follows from the embedding $\mathbf{W}_{0}^{1, p^{\prime}}(\Omega) \subset \mathbf{C}_{0}(\omega)$.

As an immediate consequence of Theorem 3.7 and Proposition 3.5 we obtain the following corollary.

COROLLARY 3.8. Under the assumptions of Theorem 3.7 and assuming that $\overline{\mathbf{u}} \neq 0$ the following statements hold,

1. If $j=j_{1}$, then $\|\bar{\varphi}\|_{\infty}=\alpha$. Moreover, if $\bar{u}_{i} \neq 0$, then $\left\|\bar{\varphi}_{i}\right\|_{\infty}=\alpha$ and

$$
\left\{\begin{array}{l}
\operatorname{Supp}\left(\bar{u}_{i}^{+}\right) \subset\left\{x \in \omega: \bar{\varphi}_{i}(x)=-\alpha\right\} \\
\operatorname{Supp}\left(\bar{u}_{i}^{-}\right) \subset\left\{x \in \omega: \bar{\varphi}_{i}(x)=+\alpha\right\} .
\end{array}\right.
$$

Copyright $@$ by SIAM. Unauthorized reproduction of this article is prohibited. 
2. If $j=j_{2}$, then $\|\bar{\varphi}\|_{2}=\alpha$. Moreover, for $i=1,2$, if $\bar{u}_{i} \neq 0$, then

$$
\left\{\begin{array}{l}
\operatorname{Supp}\left(\bar{u}_{i}^{+}\right) \subset\left\{x \in \omega: \bar{\varphi}_{i}(x)=-\left\|\bar{\varphi}_{i}\right\|_{\infty}\right\} \\
\operatorname{Supp}\left(\bar{u}_{i}^{-}\right) \subset\left\{x \in \omega: \bar{\varphi}_{i}(x)=+\left\|\bar{\varphi}_{i}\right\|_{\infty}\right\}
\end{array}\right.
$$

3. If $j=j_{\infty}$, then $\|\bar{\varphi}\|_{1}=\alpha$. Moreover, for $i=1,2$, if $\bar{u}_{i} \neq 0$, then (3.27) holds.

4. Second order optimality conditions. In this section, we prove the second order necessary and sufficient conditions for a local minimum. This will be done for the case $j=j_{1}$, i.e.,

$$
j(u)=\left\|u_{1}\right\|_{M(\omega)}+\left\|u_{2}\right\|_{M(\omega)} .
$$

Let $(\overline{\mathbf{y}}, \overline{\mathbf{u}}) \in \mathbf{W}_{0}^{1, p}(\Omega) \times \mathbf{M}(\omega)$ along with the pressure $\overline{\mathfrak{p}}$ satisfy the state equation (1.1). Throughout this section we assume that $\overline{\mathbf{y}}$ is regular, as introduced in Definition 2.7. We also assume that $\bar{\varphi} \in \mathbf{W}_{0}^{1, p^{\prime}}(\Omega)$ satisfies the first order optimality conditions (3.24) and (3.25). Then, for every $\tau \geq 0$ we consider the cones of critical directions

$$
C_{\overline{\mathbf{u}}}^{\tau}=\left\{\mathbf{v} \in \mathbf{M}(\omega): F^{\prime}(\overline{\mathbf{u}}) \mathbf{v}+\alpha j^{\prime}(\overline{\mathbf{u}} ; \mathbf{v}) \leq \tau\left\|\mathbf{z}_{\mathbf{v}}\right\|_{\mathbf{L}^{2}(\Omega)}\right\} .
$$

Observe that

$$
F^{\prime}(\overline{\mathbf{u}}) \mathbf{v}+\alpha j^{\prime}(\overline{\mathbf{u}} ; \mathbf{v}) \geq 0 \quad \forall \mathbf{v} \in \mathbf{M}(\omega) .
$$

Indeed, this follows from (3.2), (3.25), and the fact that

$$
\langle\lambda, \mathbf{v}-\overline{\mathbf{u}}\rangle \leq j^{\prime}(\overline{\mathbf{u}} ; \mathbf{v}) \quad \forall \lambda \in \partial j(\overline{\mathbf{u}}) .
$$

This inequality is consequence of the convexity and Lipschitz property of $j$. Thus, when $\tau=0$, the cone $C_{\overline{\mathbf{u}}}=C_{\overline{\mathbf{u}}}^{0}$ is given by

$$
C_{\overline{\mathbf{u}}}=\left\{\mathbf{v} \in \mathbf{M}(\omega): F^{\prime}(\overline{\mathbf{u}}) \mathbf{v}+\alpha j^{\prime}(\overline{\mathbf{u}} ; \mathbf{v})=0\right\} .
$$

Therefore, the cones $C_{\overline{\mathbf{u}}}^{\tau}$ with $\tau>0$ small can be considered as small extensions of $C_{\overline{\mathbf{u}}}$. While the necessary second order conditions will be formulated, as expected, on the cone $C_{\overline{\mathbf{u}}}$, the sufficient second order conditions require an extended cone; see, for instance, [14] and [21]. To this end, we have introduced the cones $C_{\overline{\mathbf{u}}}^{\tau}$.

Let us state some properties of $C_{\overline{\mathbf{u}}}$.

Proposition 4.1. The following statements hold.

1. $C_{\overline{\mathbf{u}}}$ is a convex cone of $\mathbf{M}(\omega)$.

2. Given $\mathbf{v}=\left(v_{1}, v_{2}\right) \in \mathbf{M}(\omega)$, we have that $\mathbf{v} \in C_{\overline{\mathbf{u}}}$ if and only if the following identities hold:

$$
\int_{\omega} \bar{\varphi}_{i} d v_{i}+\alpha j_{0}^{\prime}\left(\bar{u}_{i} ; v_{i}\right)=0 \text { for } i=1,2 .
$$

3. For every $\mathbf{v} \in \mathbf{M}(\omega)$ such that $v_{i}$ is an absolutely continuous measure with respect to $\bar{u}_{i}$ with $i=1,2$, we have that $F^{\prime}(\overline{\mathbf{u}}) \mathbf{v}+\alpha j^{\prime}(\overline{\mathbf{u}} ; \mathbf{v})=0$, hence $\mathbf{v} \in C_{\overline{\mathbf{u}}}$.

4. The following equivalent expression for $C_{\overline{\mathbf{u}}}$ holds

$$
C_{\overline{\mathbf{u}}}=\left\{\mathbf{v} \in \mathbf{M}(\omega): F^{\prime}(\overline{\mathbf{u}}) \mathbf{v}_{s}+\alpha j^{\prime}\left(\overline{\mathbf{u}} ; \mathbf{v}_{s}\right)=0\right\}
$$

with $\mathbf{v}_{s}=\left(v_{1 s}, v_{2 s}\right)$, where $v_{i s}$ is the singular part of $v_{i}$ with respect to $\left|\bar{u}_{i}\right|$, $i=1,2$. 
Proof.

1. Because of (4.1), $\mathbf{v} \in C_{\overline{\mathbf{u}}}$ if and only if $F^{\prime}(\overline{\mathbf{u}}) \mathbf{v}_{s}+\alpha j^{\prime}\left(\overline{\mathbf{u}} ; \mathbf{v}_{s}\right) \leq 0$. Then, the convexity of $C_{\overline{\mathbf{u}}}$ follows from the linearity of $F^{\prime}(\overline{\mathbf{u}})$ and the convexity of the mapping $\mathbf{v} \rightarrow j^{\prime}(\overline{\mathbf{u}} ; \mathbf{v})$.

2. Since

$$
F^{\prime}(\overline{\mathbf{u}}) \mathbf{v}+\alpha j^{\prime}(\overline{\mathbf{u}} ; \mathbf{v})=\sum_{i=1}^{2}\left(\int_{\omega} \bar{\varphi}_{i} d v_{i}+\alpha j_{0}^{\prime}\left(\bar{u}_{i} ; v_{i}\right)\right)
$$

holds, then $\mathbf{v} \in C_{\overline{\mathbf{u}}}$ if (4.2) is satisfied. For the converse implication, we take $\mathbf{v} \in C_{\overline{\mathbf{u}}}$ and apply $(4.1)$ to $\left(v_{1}, 0\right)$ and deduce that

$$
\int_{\omega} \bar{\varphi}_{1} d v_{1}+\alpha j_{0}^{\prime}\left(\bar{u}_{1} ; v_{1}\right) \geq 0
$$

Analogously we proceed with $\left(0, v_{2}\right)$ to deduce the same inequality. Adding both inequalities we infer with (4.4) that (4.2) holds.

3. From (3.20) and (3.21) we get that the mapping $\mathbf{v} \rightarrow j^{\prime}(\overline{\mathbf{u}} ; \mathbf{v})$ is linear on the space of measures $\mathbf{v}=\left(v_{1}, v_{2}\right)$ such that $v_{i}$ is absolutely continuous with respect to $\bar{u}_{i}$ for $i=1,2$. Therefore, (4.1) implies $F^{\prime}(\overline{\mathbf{u}}) \mathbf{v}+\alpha j^{\prime}(\overline{\mathbf{u}} ; \mathbf{v})=0$.

4. From (3.20), (3.21), and 3 we have that

$$
\begin{aligned}
& F^{\prime}(\overline{\mathbf{u}}) \mathbf{v}+\alpha j^{\prime}(\overline{\mathbf{u}} ; \mathbf{v})=F^{\prime}(\overline{\mathbf{u}}) \mathbf{v}_{a}+\alpha j^{\prime}\left(\overline{\mathbf{u}} ; \mathbf{v}_{a}\right) \\
& \quad+F^{\prime}(\overline{\mathbf{u}}) \mathbf{v}_{s}+\alpha j^{\prime}\left(\overline{\mathbf{u}} ; \mathbf{v}_{s}\right)=F^{\prime}(\overline{\mathbf{u}}) \mathbf{v}_{s}+\alpha j^{\prime}\left(\overline{\mathbf{u}} ; \mathbf{v}_{s}\right)
\end{aligned}
$$

where $\mathbf{v}=\mathbf{v}_{a}+\mathbf{v}_{s}$, with $v_{i a}$ the absolutely continuous part of $v_{i}$ with respect to $\left|u_{i}\right|$ and $v_{i s}$ the singular part. The above identity implies (4.3).

Next we prove the second order necessary conditions.

TheOrem 4.2. If $(\overline{\mathbf{y}}, \overline{\mathbf{u}})$ is a local solution of $(\mathrm{P})$, then $J^{\prime \prime}(\overline{\mathbf{u}}) \mathbf{v}^{2} \geq 0$ for all $\mathbf{v} \in C_{\overline{\mathbf{u}}}$ holds.

Proof. This proof follows the steps of the Theorem 3.7 in [4]. Given $\mathbf{v}=\left(v_{1}, v_{2}\right) \in$ $C_{\overline{\mathbf{u}}}$ we consider the Lebesgue decomposition $d v_{i}=g_{v_{i}} d\left|\bar{u}_{i}\right|+d v_{i s}$ for $i=1$, 2. For every integer $k \geq 1$ we set

$g_{v_{i, k}}(x)=\operatorname{Proj}_{[-k,+k]}\left(g_{v_{i}}(x)\right), d v_{i, k}=g_{v_{i, k}} d\left|\bar{u}_{i}\right|+d v_{i s}, i=1,2$, and $\mathbf{v}_{k}=\left(v_{1, k}, v_{2, k}\right)$.

Then, we have

$$
\left\|\mathbf{v}-\mathbf{v}_{k}\right\|_{\mathbf{M}(\omega)}=\sum_{i=1}^{2}\left\|g_{v_{i}}-g_{v_{i, k}}\right\|_{L^{1}\left(\left|\bar{u}_{i}\right|\right)} \rightarrow 0
$$

by Lebesgue's dominated convergence theorem. Moreover, since the singular parts of $v_{i, k}$ and $v_{i}$ coincide for $i=1,2$, and $\mathbf{v} \in C_{\overline{\mathbf{u}}}$, then (4.3) implies that $\mathbf{v}_{k} \in C_{\bar{u}}$ for every $k$.

Next let us express $d \bar{u}_{i}=h_{i} d\left|\bar{u}_{i}\right|$, where $h_{i}$ is measurable with respect to $\left|\bar{u}_{i}\right|$ and $\left|h_{i}(x)\right|=1$ for all $x \in \omega, d \bar{u}_{i}^{+}=h_{i}^{+} d\left|\bar{u}_{i}\right|$ and $d \bar{u}_{i}^{-}=h_{i}^{-} d\left|\bar{u}_{i}\right|$, where $\bar{u}_{i}=\bar{u}_{i}^{+}-\bar{u}_{i}^{-}$ is the Jordan decomposition of $\bar{u}_{i}$, for $i=1,2$. See, for instance, [23, Chapter 6] for details. Let us define the sets

$$
\omega_{i}^{+}=\left\{x \in \omega: h_{i}(x)=+1\right\} \text { and } \omega_{i}^{-}=\left\{x \in \omega: h_{i}(x)=-1\right\}, \quad i=1,2 .
$$

Copyright (c) by SIAM. Unauthorized reproduction of this article is prohibited. 
For any $0<\rho<\frac{1}{k}$ we have for $i=1,2$

$$
\begin{aligned}
& \frac{j_{0}\left(\bar{u}_{i}+\rho v_{i, k}\right)-j_{0}\left(\bar{u}_{i}\right)}{\rho} \\
& \quad=\int_{\omega_{i}^{+}} \frac{\left|1+\rho g_{v_{i, k}}\right|-1}{\rho} d \bar{u}_{i}^{+}+\int_{\omega_{i}^{-}} \frac{\left|-1+\rho g_{v_{i, k}}\right|-1}{\rho} d \bar{u}_{i}^{-}+\left\|v_{i s}\right\|_{M(\omega)} \\
& \quad=\int_{\omega} g_{v_{i, k}} d \bar{u}_{i}+\left\|v_{i s}\right\|_{M(\omega)}=j_{0}^{\prime}\left(\bar{u}_{i} ; v_{i, k}\right) .
\end{aligned}
$$

Using that $\overline{\mathbf{u}}$ is a local minimum of $J$ and making a Taylor expansion of $F$ we get for every $k$ and $0<\rho<\frac{1}{k}$ the existence of $\theta=\theta(k, \rho)$, with $0<\theta<1$, such that

$$
\begin{aligned}
0 & \leq \frac{J\left(\overline{\mathbf{u}}+\rho \mathbf{v}_{k}\right)-J(\overline{\mathbf{u}})}{\rho} \\
& =F^{\prime}(\overline{\mathbf{u}}) \mathbf{v}_{k}+\frac{\rho}{2} F^{\prime \prime}\left(\overline{\mathbf{u}}+\theta \rho \mathbf{v}_{k}\right) \mathbf{v}_{k}^{2}+\alpha j^{\prime}\left(\overline{\mathbf{u}} ; \mathbf{v}_{k}\right)=\frac{\rho}{2} F^{\prime \prime}\left(\overline{\mathbf{u}}+\theta \rho \mathbf{v}_{k}\right) \mathbf{v}_{k}^{2},
\end{aligned}
$$

since $\mathbf{v}_{k} \in C_{\overline{\mathbf{u}}}$. Finally, dividing the last term by $\rho / 2$ and taking the limit when $k \rightarrow \infty$, we get that $F^{\prime \prime}(\overline{\mathbf{u}}) \mathbf{v}^{2} \geq 0$.

Now we formulate our second order condition for local optimality:

$$
\exists \tau>0 \text { and } \exists \delta>0: F^{\prime \prime}(\overline{\mathbf{u}}) \mathbf{v}^{2} \geq \delta\left\|\mathbf{z}_{\mathbf{v}}\right\|_{\mathbf{L}^{2}(\Omega)}^{2} \quad \forall \mathbf{v} \in C_{\mathbf{u}}^{\tau} .
$$

TheOREM 4.3. Let $\overline{\mathbf{u}}$ satisfy the first order optimality conditions (3.24)-(3.25), and assume that (4.5) holds. Then, there exist $\varepsilon>0$ and $\kappa>0$ such that

$$
J(\overline{\mathbf{u}})+\frac{\kappa}{2}\left\|\mathbf{y}_{\mathbf{u}}-\overline{\mathbf{y}}\right\|_{\mathbf{L}^{2}(\Omega)}^{2} \leq J(\mathbf{u}), \forall \mathbf{u} \in \mathbf{M}(\omega) \text { such that }\|\mathbf{u}-\overline{\mathbf{u}}\|_{\mathbf{w}^{-1, p}(\Omega)} \leq \varepsilon
$$

We will establish some lemmas required for the proof of this theorem. According to Theorem 2.10, $\mathcal{N}_{p}$ can be chosen bounded and convex and such that $\mathcal{G}^{\prime}(\mathbf{u})$ : $\mathbf{W}^{-1, p}(\Omega) \longrightarrow \mathbf{W}_{p} \times L^{p}(\Omega) / \mathbb{R}$ is an isomorphism and

$$
\exists M_{\mathcal{G}} \text { such that }\left\|\mathcal{G}^{\prime}(\mathbf{f})\right\|_{\mathcal{L}\left(\mathbf{W}^{-1, p}(\Omega), \mathbf{W}_{p} \times L^{p}(\Omega) / \mathbb{R}\right)} \leq M_{\mathcal{G}} \quad \forall \mathbf{f} \in \mathcal{N}_{p} .
$$

Following the notation introduced in Corollary 2.11, we set $\mathcal{U}_{p}=\iota^{-1}\left(\mathcal{N}_{p}\right)=$ $\left(\mathcal{N}_{p}-\mathbf{f}_{0}\right) \cap \mathbf{M}(\omega)$, which is also an open, bounded, and convex subset of $\mathbf{M}(\omega)$.

LEMma 4.4. The following inequality holds:

$$
\left\|\mathbf{y}_{\mathbf{u}}-\overline{\mathbf{y}}\right\|_{\mathbf{W}_{0}^{1, p}(\Omega)} \leq M_{\mathcal{G}}\|\mathbf{u}-\overline{\mathbf{u}}\|_{\mathbf{W}^{-1, p}(\Omega)} \quad \forall \mathbf{u} \in \mathcal{U}_{p}
$$

Proof. This is an immediate consequence of the mean value theorem and (4.7).

Lemma 4.5. Given $\mathbf{u} \in \mathcal{U}_{p}$ and $\mathbf{v} \in \mathbf{M}(\omega)$, we set $\mathbf{z}_{\mathbf{u}, \mathbf{v}}=\mathcal{G}^{\prime}\left(\mathbf{f}_{0}+\chi_{\omega} \mathbf{u}\right) \chi_{\omega} \mathbf{v}$ and $\mathbf{z}_{\mathbf{v}}=\mathcal{G}^{\prime}\left(\mathbf{f}_{0}+\chi_{\omega} \overline{\mathbf{u}}\right) \chi_{\omega} \mathbf{v}$. Then there exist constants $M_{1}>0$ and $M_{2}>0$ independent of $\mathbf{u}$ and $\mathbf{v}$ such that

$$
\begin{aligned}
& \left\|\mathbf{z}_{\mathbf{u}, \mathbf{v}}-\mathbf{z}_{\mathbf{V}}\right\|_{\mathbf{W}_{0}^{1, p}(\Omega)} \leq M_{1}\|\mathbf{u}-\overline{\mathbf{u}}\|_{\mathbf{W}^{-1, p}(\Omega)}\left\|\mathbf{z}_{\mathbf{v}}\right\|_{\mathbf{L}^{2}(\Omega)}, \\
& \left\|\mathbf{z}_{\mathbf{u}, \mathbf{v}}\right\|_{\mathbf{L}^{2}(\Omega)} \leq M_{2}\left\|\mathbf{z}_{\mathbf{V}}\right\|_{\mathbf{L}^{2}(\Omega)} .
\end{aligned}
$$

Copyright $@$ by SIAM. Unauthorized reproduction of this article is prohibited. 
Proof. According to (2.20), the equations satisfied by $\mathbf{z}_{\mathbf{u}, \mathbf{v}}$ and $\mathbf{z}_{\mathbf{v}}$ are

$$
\begin{aligned}
& -\nu \Delta \mathbf{z}_{\mathbf{u}, \mathbf{v}}+\left(\mathbf{y}_{\mathbf{u}} \cdot \nabla\right) \mathbf{z}_{\mathbf{u}, \mathbf{v}}+\left(\mathbf{z}_{\mathbf{u}, \mathbf{v}} \cdot \nabla\right) \mathbf{y}_{\mathbf{u}}+\mathfrak{q}_{\mathbf{u}}=\chi_{\omega} \mathbf{v}, \\
& -\nu \Delta \mathbf{z}_{\mathbf{v}}+(\overline{\mathbf{y}} \cdot \nabla) \mathbf{z}_{\mathbf{v}}+\left(\mathbf{z}_{\mathbf{v}} \cdot \nabla\right) \overline{\mathbf{y}}+\overline{\mathfrak{q}}=\chi_{\omega} \mathbf{v} .
\end{aligned}
$$

Subtracting both equations and setting $\mathbf{e}=\mathbf{z}_{\mathbf{u}, \mathbf{v}}-\mathbf{z}_{\mathbf{v}}$ and $\mathfrak{q}=\mathfrak{q}_{\mathbf{v}}-\overline{\mathfrak{q}}$ we get

$$
-\nu \Delta \mathbf{e}+\left(\mathbf{y}_{\mathbf{u}} \cdot \nabla\right) \mathbf{e}+(\mathbf{e} \cdot \nabla) \mathbf{y}_{\mathbf{u}}+\mathfrak{q}=\mathbf{g},
$$

where $\mathbf{g}=-\left[\left(\mathbf{y}_{\mathbf{u}}-\overline{\mathbf{y}}\right) \cdot \nabla\right] \mathbf{z}_{\mathbf{v}}-\left(\mathbf{z}_{\mathbf{v}} \cdot \nabla\right)\left(\mathbf{y}_{\mathbf{u}}-\overline{\mathbf{y}}\right)$. Let us estimate $\mathbf{g}$ in $\mathbf{W}^{-1, p}(\Omega)$. We prove the estimate for the first term, the second being identical. For arbitrary $\mathbf{w} \in \mathbf{W}_{0}^{1, p^{\prime}}(\Omega)$ we have

$$
\begin{aligned}
& \left|\int_{\Omega}\left[\left(\mathbf{y}_{\mathbf{u}}-\overline{\mathbf{y}}\right) \cdot \nabla\right] \mathbf{z}_{\mathbf{v}} \mathbf{w} d x\right|=\left|\int_{\Omega}\left[\left(\mathbf{y}_{\mathbf{u}}-\overline{\mathbf{y}}\right) \cdot \nabla\right] \mathbf{w} \mathbf{z}_{\mathbf{v}} d x\right| \\
& \quad \leq\left\|\mathbf{z}_{\mathbf{v}}\right\|_{\mathbf{L}^{2}(\Omega)}\left\|\mathbf{y}_{\mathbf{u}}-\overline{\mathbf{y}}\right\|_{\mathbf{L}^{\frac{2 p}{2-p}(\Omega)}}\|\nabla \mathbf{w}\|_{L^{p^{\prime}}(\Omega) / \mathbb{R}} \\
& \quad \leq C_{\Omega, p}\left\|\mathbf{z}_{\mathbf{v}}\right\|_{\mathbf{L}^{2}(\Omega)}\left\|\mathbf{y}_{\mathbf{u}}-\overline{\mathbf{y}}\right\|_{\mathbf{W}_{0}^{1, p}(\Omega)}\|\nabla \mathbf{w}\|_{\mathbf{L}^{p^{\prime}}(\Omega)} .
\end{aligned}
$$

Then, we obtain

$$
\|\mathbf{g}\|_{\mathbf{W}^{-1, p}(\Omega)} \leq 2 C_{\Omega, p}\left\|\mathbf{z}_{\mathbf{V}}\right\|_{\mathbf{L}^{2}(\Omega)}\left\|\mathbf{y}_{\mathbf{u}}-\overline{\mathbf{y}}\right\|_{\mathbf{W}_{0}^{1, p}(\Omega)} .
$$

Taking into account that $(\mathbf{e}, \mathfrak{q})=\mathcal{G}^{\prime}\left(\mathbf{f}_{0}+\chi_{\omega} \mathbf{u}\right) \mathbf{g}$, from (4.7) and (4.8) we infer

$$
\begin{aligned}
& \|\mathbf{e}\|_{\mathbf{W}_{0}^{1, p}(\Omega)} \leq 2 M_{\mathcal{G}} C_{\Omega, p}\left\|\mathbf{z}_{\mathbf{v}}\right\|_{\mathbf{L}^{2}(\Omega)}\left\|\mathbf{y}_{\mathbf{u}}-\overline{\mathbf{y}}\right\|_{\mathbf{W}_{0}^{1, p}(\Omega)} \\
& \quad \leq 2 M_{\mathcal{G}}^{2} C_{\Omega, p}\left\|\mathbf{z}_{\mathbf{v}}\right\|_{\mathbf{L}^{2}(\Omega)}\|\mathbf{u}-\overline{\mathbf{u}}\|_{\mathbf{W}^{-1, p}(\Omega)} .
\end{aligned}
$$

Hence, (4.9) holds with $M_{1}=2 M_{\mathcal{G}}^{2} C_{\Omega, p}$. Finally, with the triangle inequality and (4.9) we deduce

$$
\begin{aligned}
& \left\|\mathbf{z}_{\mathbf{u}, \mathbf{v}}\right\|_{\mathbf{L}^{2}(\Omega)} \leq\left\|\mathbf{z}_{\mathbf{u}, \mathbf{v}}-\mathbf{z}_{\mathbf{V}}\right\|_{\mathbf{L}^{2}(\Omega)}+\left\|\mathbf{z}_{\mathbf{v}}\right\|_{\mathbf{L}^{2}(\Omega)} \leq C_{\Omega, p}^{\prime}\left\|\mathbf{z}_{\mathbf{u}, \mathbf{v}}-\mathbf{z}_{\mathbf{v}}\right\|_{\mathbf{W}_{0}^{1, p}(\Omega)}+\left\|\mathbf{z}_{\mathbf{V}}\right\|_{\mathbf{L}^{2}(\Omega)} \\
& \quad \leq\left(C_{\Omega, p}^{\prime} M_{1}\|\mathbf{u}-\overline{\mathbf{u}}\|_{\mathbf{W}^{-1, p}(\Omega)}+1\right)\left\|\mathbf{z}_{\mathbf{V}}\right\|_{\mathbf{L}^{2}(\Omega)} .
\end{aligned}
$$

Inequality (4.10) follows from the above inequality using the boundedness of $\mathcal{U}_{p}$.

Lemma 4.6. There exist $\varepsilon_{1}>0$ and $M_{3}>0$ such that

$$
\left\|\mathbf{y}_{\mathbf{u}}-\overline{\mathbf{y}}\right\|_{\mathbf{L}^{2}(\Omega)} \leq M_{3}\left\|\mathbf{z}_{\mathbf{u}-\overline{\mathbf{u}}}\right\|_{\mathbf{L}^{2}(\Omega)} \forall \mathbf{u} \in \mathcal{U}_{p} \text { with }\|\mathbf{u}-\overline{\mathbf{u}}\|_{\mathbf{W}^{-1, p}(\Omega)} \leq \varepsilon_{1},
$$

where $\mathbf{z}_{\mathbf{u}-\overline{\mathbf{u}}}=\mathcal{G}^{\prime}\left(\mathbf{f}_{0}+\chi_{\omega} \overline{\mathbf{u}}\right)\left(\chi_{\omega}(\mathbf{u}-\overline{\mathbf{u}})\right)$.

Proof. Let us consider the equations satisfied by $\mathbf{y}_{\mathbf{u}}, \overline{\mathbf{y}}$ and $\mathbf{z}_{\mathbf{u}-\overline{\mathbf{u}}}$ :

$$
\begin{aligned}
& -\nu \Delta \mathbf{y}_{\mathbf{u}}+\left(\mathbf{y}_{\mathbf{u}} \cdot \nabla\right) \mathbf{y}_{\mathbf{u}}+\nabla \mathfrak{p}_{\mathbf{u}}=\chi_{\omega} \mathbf{u}, \\
& -\nu \Delta \overline{\mathbf{y}}+(\overline{\mathbf{y}} \cdot \nabla) \overline{\mathbf{y}}+\nabla \overline{\mathfrak{p}}=\chi_{\omega} \overline{\mathbf{u}}, \\
& -\nu \Delta \mathbf{z}_{\mathbf{u}-\overline{\mathbf{u}}}+(\overline{\mathbf{y}} \cdot \nabla) \mathbf{z}_{\mathbf{u}-\overline{\mathbf{u}}}+\left(\mathbf{z}_{\mathbf{u}-\overline{\mathbf{u}}} \cdot \nabla\right) \overline{\mathbf{y}}+\nabla \mathfrak{q}_{\mathbf{u}-\overline{\mathbf{u}}}=\chi_{\omega}(\mathbf{u}-\overline{\mathbf{u}}) .
\end{aligned}
$$

Setting $\mathbf{e}=\mathbf{y}_{\mathbf{u}}-\overline{\mathbf{y}}-\mathbf{z}_{\mathbf{u}-\overline{\mathbf{u}}}$ and $\mathfrak{q}=\mathfrak{p}_{u}-\overline{\mathfrak{p}}-\mathfrak{q}_{\mathbf{u}-\overline{\mathbf{u}}}$, we infer from the above equations

$$
-\nu \Delta \mathbf{e}+(\overline{\mathbf{y}} \cdot \nabla) \mathbf{e}+(\mathbf{e} \cdot \nabla) \overline{\mathbf{y}}+\nabla \mathfrak{q}=-\left[\left(\mathbf{y}_{\mathbf{u}}-\overline{\mathbf{y}}\right) \cdot \nabla\right]\left(\mathbf{y}_{\mathbf{u}}-\overline{\mathbf{y}}\right) .
$$

Copyright $@$ by SIAM. Unauthorized reproduction of this article is prohibited. 
Arguing as in the previous lemma, we can prove that $\left[\left(\mathbf{y}_{\mathbf{u}}-\overline{\mathbf{y}}\right) \cdot \nabla\right]\left(\mathbf{y}_{\mathbf{u}}-\overline{\mathbf{y}}\right) \in \mathbf{W}^{-1, p}(\Omega)$, and with (4.8)

$$
\begin{aligned}
& \left\|\left[\left(\mathbf{y}_{\mathbf{u}}-\overline{\mathbf{y}}\right) \cdot \nabla\right]\left(\mathbf{y}_{\mathbf{u}}-\overline{\mathbf{y}}\right)\right\|_{\mathbf{W}^{-1, p}(\Omega)} \leq C_{\Omega, p}\left\|\mathbf{y}_{\mathbf{u}}-\overline{\mathbf{y}}\right\|_{\mathbf{L}^{2}(\Omega)}\left\|\mathbf{y}_{\mathbf{u}}-\overline{\mathbf{y}}\right\|_{\mathbf{W}_{0}^{1, p}(\Omega)} \\
& \quad \leq C_{\Omega, p} M_{\mathcal{G}}\|\mathbf{u}-\overline{\mathbf{u}}\|_{\mathbf{W}^{-1, p}(\Omega)}\left\|\mathbf{y}_{\mathbf{u}}-\overline{\mathbf{y}}\right\|_{\mathbf{L}^{2}(\Omega)} \leq C_{\Omega, p} M_{\mathcal{G}} \varepsilon_{1}\left\|\mathbf{y}_{\mathbf{u}}-\overline{\mathbf{y}}\right\|_{\mathbf{L}^{2}(\Omega)}
\end{aligned}
$$

Now, using that $(\mathbf{e}, \mathfrak{q})=\mathcal{G}^{\prime}\left(\mathbf{f}_{0}+\chi_{\omega} \overline{\mathbf{u}}\right)\left(-\left[\left(\mathbf{y}_{\mathbf{u}}-\overline{\mathbf{y}}\right) \cdot \nabla\right]\left(\mathbf{y}_{\mathbf{u}}-\overline{\mathbf{y}}\right)\right)$ and (4.7) we get

$$
\|\mathbf{e}\|_{\mathbf{L}^{2}(\Omega)} \leq C_{\Omega, p}^{\prime}\|\mathbf{e}\|_{\mathbf{W}_{0}^{1, p}(\Omega)} \leq C_{\Omega, p} C_{\Omega, p}^{\prime} M_{\mathcal{G}}^{2} \varepsilon_{1}\left\|\mathbf{y}_{\mathbf{u}}-\overline{\mathbf{y}}\right\|_{\mathbf{L}^{2}(\Omega)} .
$$

With $\left\|\mathbf{y}_{\mathbf{u}}-\overline{\mathbf{y}}\right\|_{\mathbf{L}^{2}(\Omega)} \leq\|\mathbf{e}\|_{\mathbf{L}^{2}(\Omega)}+\left\|\mathbf{z}_{\mathbf{u}-\overline{\mathbf{u}}}\right\|_{\mathbf{L}^{2}(\Omega)}$ and taking

$$
0<\varepsilon_{1}<\frac{1}{C_{\Omega, p}^{\prime} C_{\Omega, p} M_{\mathcal{G}}^{2}} \text { and } M_{3}=\frac{1}{1-C_{\Omega, p} C_{\Omega, p}^{\prime} M_{\mathcal{G}}^{2} \varepsilon_{1}}
$$

(4.12) follows.

LEMMA 4.7. There exists a constant $M_{4}>0$ such that

$$
\left\|\boldsymbol{\varphi}_{\mathbf{u}}-\bar{\varphi}\right\|_{\mathbf{W}_{0}^{1, p^{\prime}}(\Omega)} \leq M_{4}\left\|\mathbf{y}_{\mathbf{u}}-\overline{\mathbf{y}}\right\|_{\mathbf{L}^{2}(\Omega)} \quad \forall \mathbf{u} \in \mathcal{U}_{p}
$$

Proof. Let us write the equations satisfied by $\varphi_{\mathbf{u}}$ and $\bar{\varphi}$

$$
\begin{aligned}
& -\nu \Delta \boldsymbol{\varphi}_{\mathbf{u}}-\left(\mathbf{y}_{\mathbf{u}} \cdot \nabla\right) \boldsymbol{\varphi}_{\mathbf{u}}+\left(\nabla \mathbf{y}_{\mathbf{u}}\right)^{T} \boldsymbol{\varphi}_{\mathbf{u}}+\nabla \pi_{\mathbf{u}}=\mathbf{y}_{\mathbf{u}}-\mathbf{y}_{d} \\
& -\nu \Delta \overline{\boldsymbol{\varphi}}-(\overline{\mathbf{y}} \cdot \nabla) \bar{\varphi}+(\nabla \overline{\mathbf{y}})^{T} \overline{\boldsymbol{\varphi}}+\nabla \bar{\pi}=\overline{\mathbf{y}}-\mathbf{y}_{d} .
\end{aligned}
$$

Now, setting $\mathbf{e}=\varphi_{\mathbf{u}}-\bar{\varphi}$ and $\mathfrak{q}=\pi_{\mathbf{u}}-\bar{\pi}$, and subtracting the above equations we infer

$$
-\nu \Delta \mathbf{e}-(\overline{\mathbf{y}} \cdot \nabla) \mathbf{e}+(\nabla \overline{\mathbf{y}})^{T} \mathbf{e}+\nabla \mathfrak{q}=\left(\mathbf{y}_{\mathbf{u}}-\overline{\mathbf{y}}\right)+\left[\left(\mathbf{y}_{\mathbf{u}}-\overline{\mathbf{y}}\right) \cdot \nabla\right] \boldsymbol{\varphi}_{\mathbf{u}}-\left(\nabla \mathbf{y}_{\mathbf{u}}-\nabla \overline{\mathbf{y}}\right)^{T} \boldsymbol{\varphi}_{\mathbf{u}}
$$

We can estimate $\mathbf{e}$ in $\mathbf{W}_{0}^{1, p^{\prime}}(\Omega)$ by using (2.19). To this end, we need to estimate the three terms of the right-hand side of the above equation in $\mathbf{W}^{-1, p^{\prime}}(\Omega)$. Moreover, to prove (4.13), this estimate should be obtained in terms of $\left\|\mathbf{y}_{\mathbf{u}}-\overline{\mathbf{y}}\right\|_{\mathbf{L}^{2}(\Omega)}$. For the first term it is enough to observe that $\mathbf{W}_{0}^{1, p}(\Omega) \subset \mathbf{L}^{2}(\Omega)$ and, hence, $\mathbf{L}^{2}(\Omega) \subset \mathbf{W}^{-1, p^{\prime}}(\Omega)$. To estimate the second term we take an arbitrary function $\mathbf{w} \in \mathbf{W}_{0}^{1, p}(\Omega)$ and proceed as follows

$$
\begin{aligned}
& \left|\int_{\Omega}\left[\left(\mathbf{y}_{\mathbf{u}}-\overline{\mathbf{y}}\right) \cdot \nabla\right] \boldsymbol{\varphi}_{\mathbf{u}} \mathbf{w} d x\right| \leq\left\|\mathbf{y}_{\mathbf{u}}-\overline{\mathbf{y}}\right\|_{\mathbf{L}^{2}(\Omega)}\left\|\nabla \boldsymbol{\varphi}_{\mathbf{u}}\right\|_{\mathbf{L}^{p^{\prime}}(\Omega)}\|\mathbf{w}\|_{\mathbf{L}^{\frac{2 p}{2-p}}(\Omega)} \\
& \quad \leq C_{\Omega, p}\left\|\mathbf{y}_{\mathbf{u}}-\overline{\mathbf{y}}\right\|_{\mathbf{L}^{2}(\Omega)}\left\|\boldsymbol{\varphi}_{\mathbf{u}}\right\|_{\mathbf{W}_{0}^{1, p^{\prime}}(\Omega)}\|\mathbf{w}\|_{\mathbf{W}_{0}^{1, p}(\Omega)} .
\end{aligned}
$$

Using (3.5) and (4.8) we deduce that $\left\|\boldsymbol{\varphi}_{\mathbf{u}}\right\|_{\mathbf{W}_{0}^{1, p^{\prime}}(\Omega)} \leq C_{2}$ for all $\mathbf{u} \in \mathcal{U}_{p}$. Therefore, we conclude that

$$
\left\|\left[\left(\mathbf{y}_{\mathbf{u}}-\overline{\mathbf{y}}\right) \cdot \nabla\right] \boldsymbol{\varphi}_{\mathbf{u}}\right\|_{\mathbf{W}^{-1, p^{\prime}}(\Omega)} \leq C_{\Omega, p} C_{2}\left\|\mathbf{y}_{\mathbf{u}}-\overline{\mathbf{y}}\right\|_{\mathbf{L}^{2}(\Omega)} .
$$

For the last term we observe that

$$
\int_{\Omega}\left(\nabla \mathbf{y}_{\mathbf{u}}-\nabla \overline{\mathbf{y}}\right)^{T} \boldsymbol{\varphi}_{\mathbf{u}} \mathbf{w} d x=-\int_{\Omega}(\mathbf{w} \cdot \nabla) \boldsymbol{\varphi}_{\mathbf{u}}\left(\mathbf{y}_{\mathbf{u}}-\overline{\mathbf{y}}\right) d x
$$

and then we obtained the same estimate as for the second term. Finally, as mentioned above, (4.13) follows from (2.19) applied to (4.14) and the obtained estimates. 
Lemma 4.8. For every $\rho>0$ there exists $\varepsilon_{\rho}>0$ such that

$$
\left|\left[F^{\prime \prime}(\mathbf{u})-F^{\prime \prime}(\overline{\mathbf{u}})\right](\mathbf{u}-\overline{\mathbf{u}})^{2}\right| \leq \rho\left\|\mathbf{z}_{\mathbf{u}-\overline{\mathbf{u}}}\right\|_{\mathbf{L}^{2}(\Omega)}^{2} \quad \forall \mathbf{u} \in B_{\varepsilon_{\rho}}(\overline{\mathbf{u}}),
$$

where $B_{\varepsilon_{\rho}}(\overline{\mathbf{u}})=\left\{\mathbf{u} \in \mathcal{U}_{p}:\|\mathbf{u}-\overline{\mathbf{u}}\|_{\mathbf{W}^{-1, p}(\Omega)} \leq \varepsilon_{\rho}\right\}$.

Proof. Let us denote $\mathbf{v}=\mathbf{u}-\overline{\mathbf{u}}, \mathbf{z}_{\mathbf{u}, \mathbf{v}}=\mathcal{G}^{\prime}\left(\mathbf{f}_{0}+\chi_{\omega} \mathbf{u}\right) \chi_{\omega} \mathbf{v}$ and $\mathbf{z}_{\mathbf{v}}=\mathcal{G}^{\prime}\left(\mathbf{f}_{0}+\right.$ $\left.\chi_{\omega} \overline{\mathbf{u}}\right) \chi_{\omega} \mathbf{v}$. According to (3.3) we have

$$
\begin{aligned}
& \left|\left[F^{\prime \prime}(\mathbf{u})-F^{\prime \prime}(\overline{\mathbf{u}})\right] \mathbf{v}^{2}\right| \\
& =\left|\int_{\Omega}\left[\left|\mathbf{z}_{\mathbf{u}, \mathbf{v}}\right|^{2}-2\left(\mathbf{z}_{\mathbf{u}, \mathbf{v}} \cdot \nabla\right) \mathbf{z}_{\mathbf{u}, \mathbf{v}} \boldsymbol{\varphi}_{\mathbf{u}}\right] d x-\int_{\Omega}\left[\left|\mathbf{z}_{\mathbf{v}}\right|^{2}-2\left(\mathbf{z}_{\mathbf{v}} \cdot \nabla\right) \mathbf{z}_{\mathbf{v}} \overline{\boldsymbol{\varphi}}\right] d x\right| \\
& \leq \int_{\Omega}\left|\mathbf{z}_{\mathbf{u}, \mathbf{v}}+\mathbf{z}_{\mathbf{v}}\right|\left|\mathbf{z}_{\mathbf{u}, \mathbf{v}}-\mathbf{z}_{\mathbf{v}}\right| d x+2\left|\int_{\Omega}\left[\left(\mathbf{z}_{\mathbf{u}, \mathbf{v}}-\mathbf{z}_{\mathbf{v}}\right) \cdot \nabla\right] \boldsymbol{\varphi}_{\mathbf{u}} \mathbf{z}_{\mathbf{u}, \mathbf{v}} d x\right| \\
& \quad+2\left|\int_{\Omega}\left(\mathbf{z}_{\mathbf{v}} \cdot \nabla\right)\left(\boldsymbol{\varphi}_{\mathbf{u}}-\overline{\boldsymbol{\varphi}}\right) \mathbf{z}_{\mathbf{u}, \mathbf{v}} d x\right|+2\left|\int_{\Omega}\left(\mathbf{z}_{\mathbf{v}} \cdot \nabla\right) \overline{\boldsymbol{\varphi}}\left(\mathbf{z}_{\mathbf{u}, \mathbf{v}}-\mathbf{z}_{\mathbf{v}}\right) d x\right| .
\end{aligned}
$$

We proceed to estimate the last four integrals. For the first one we use Lemma 4.5 as follows

$$
\begin{aligned}
& \int_{\Omega}\left|\mathbf{z}_{\mathbf{u}, \mathbf{v}}+\mathbf{z}_{\mathbf{v}}\right|\left|\mathbf{z}_{\mathbf{u}, \mathbf{v}}-\mathbf{z}_{\mathbf{v}}\right| d x \leq\left\|\mathbf{z}_{\mathbf{u}, \mathbf{v}}+\mathbf{z}_{\mathbf{v}}\right\|_{\mathbf{L}^{2}(\Omega)}\left\|\mathbf{z}_{\mathbf{u}, \mathbf{v}}-\mathbf{z}_{\mathbf{v}}\right\|_{\mathbf{L}^{2}(\Omega)} \\
& \quad \leq C_{\Omega, p}^{\prime}\left(1+M_{2}\right) M_{1}\|\mathbf{u}-\overline{\mathbf{u}}\|_{\mathbf{W}^{-1, p}(\Omega)}\left\|\mathbf{z}_{\mathbf{v}}\right\|_{\mathbf{L}^{2}(\Omega)}^{2}
\end{aligned}
$$

Let us estimate the second integral. First, we observe that, as pointed out in the proof of Lemma 4.7 , there exists a constant $C_{2}$ such that $\left\|\varphi_{\mathbf{u}}\right\|_{\mathbf{W}_{0}^{1, p^{\prime}}(\Omega)} \leq C_{2}$ for every $\mathbf{u} \in \mathcal{U}_{p}$. Using again Lemma 4.5 and this inequality we get

$$
\begin{aligned}
& \left|\int_{\Omega}\left[\left(\mathbf{z}_{\mathbf{u}, \mathbf{v}}-\mathbf{z}_{\mathbf{v}}\right) \cdot \nabla\right] \boldsymbol{\varphi}_{\mathbf{u}} \mathbf{z}_{\mathbf{u}, \mathbf{v}} d x\right| \leq\left\|\left(\mathbf{z}_{\mathbf{u}, \mathbf{v}}-\mathbf{z}_{\mathbf{v}}\right) \mathbf{z}_{\mathbf{u}, \mathbf{v}}\right\|_{\mathbf{L}^{p}(\Omega)}\left\|\nabla \boldsymbol{\varphi}_{\mathbf{u}}\right\|_{\mathbf{L}^{p^{\prime}}(\Omega)} \\
& \quad \leq C_{2}\left\|\mathbf{z}_{\mathbf{u}, \mathbf{v}}-\mathbf{z}_{\mathbf{v}}\right\|_{\mathbf{L}^{\frac{2 p}{2-p}}(\Omega)}\left\|\mathbf{z}_{\mathbf{u}, \mathbf{v}}\right\|_{\mathbf{L}^{2}(\Omega)} \leq C_{2} C_{\Omega, p}\left\|\mathbf{z}_{\mathbf{u}, \mathbf{v}}-\mathbf{z}_{\mathbf{v}}\right\|_{\mathbf{W}_{0}^{1, p}(\Omega)}\left\|\mathbf{z}_{\mathbf{u}, \mathbf{v}}\right\|_{\mathbf{L}^{2}(\Omega)} \\
& \quad \leq C_{2} C_{\Omega, p} M_{1} M_{2}\|\mathbf{u}-\overline{\mathbf{u}}\|_{\mathbf{W}^{-1, p}(\Omega)}\left\|\mathbf{z}_{\mathbf{v}}\right\|_{\mathbf{L}^{2}(\Omega)}^{2}
\end{aligned}
$$

For the third integral we select $\varepsilon_{1}>0$ so that (4.12) holds. Moreover, we observe that (4.7) implies

$$
\left\|\mathbf{z}_{\mathbf{v}}\right\|_{\mathbf{W}_{0}^{1, p}(\Omega)} \leq M_{\mathcal{G}}\|\mathbf{v}\|_{\mathbf{W}^{-1, p}(\Omega)}=M_{\mathcal{G}}\|\mathbf{u}-\overline{\mathbf{u}}\|_{\mathbf{W}^{-1, p}(\Omega)} .
$$

Then, with (4.10), (4.12), (4.13), (4.18), and recalling that $\mathbf{v}=\mathbf{u}-\overline{\mathbf{u}}$ we obtain

$$
\begin{aligned}
& \left|\int_{\Omega}\left(\mathbf{z}_{\mathbf{v}} \cdot \nabla\right)\left(\boldsymbol{\varphi}_{\mathbf{u}}-\overline{\boldsymbol{\varphi}}\right) \mathbf{z}_{\mathbf{u}, \mathbf{v}} d x\right| \leq\left\|\left|\mathbf{z}_{\mathbf{u}, \mathbf{v}}\right|\left|\mathbf{z}_{\mathbf{v}}\right|\right\|_{\mathbf{L}^{p}(\Omega)}\left\|\boldsymbol{\varphi}_{\mathbf{u}}-\overline{\boldsymbol{\varphi}}\right\|_{\mathbf{W}_{0}^{1, p^{\prime}}(\Omega)} \\
& \quad M_{4}\left\|\mathbf{z}_{\mathbf{u}, \mathbf{v}}\right\|_{\mathbf{L}^{2}(\Omega)}\left\|\mathbf{z}_{\mathbf{v}}\right\|_{\mathbf{L}^{\frac{2 p}{2-p}}(\Omega)}\left\|\mathbf{y}_{\mathbf{u}}-\overline{\mathbf{y}}\right\|_{\mathbf{L}^{2}(\Omega)} \leq C_{\Omega, p} M_{2} M_{3} M_{4}\left\|z_{\mathbf{v}}\right\|_{\mathbf{W}_{0}^{1, p}(\Omega)}\left\|\mathbf{z}_{\mathbf{V}}\right\|_{\mathbf{L}^{2}(\Omega)}^{2} \\
& \quad \leq C_{\Omega, p} M_{2} M_{3} M_{4} M_{\mathcal{G}}\|\mathbf{u}-\overline{\mathbf{u}}\|_{\mathbf{W}^{-1, p}(\Omega)}\left\|\mathbf{z}_{\mathbf{v}}\right\|_{\mathbf{L}^{2}(\Omega)}^{2} .
\end{aligned}
$$

Finally, the estimation for the fourth integral is the same as the one proved for the second integral just replacing $C_{2}$ by $\|\bar{\varphi}\|_{\mathbf{W}_{0}^{1, p^{\prime}}(\Omega)}$. From the obtained estimates for the integrals the existence of $\varepsilon_{\rho} \in\left(0, \varepsilon_{1}\right)$ such that (4.15) holds is immediate.

Copyright $\odot$ by SIAM. Unauthorized reproduction of this article is prohibited. 
Proof of Theorem 4.3. Let us first estimate $\left|F^{\prime \prime}(\mathbf{u})(\mathbf{u}-\overline{\mathbf{u}})^{2}\right|$. To this end, we recall the existence of a constant $C_{2}>0$ such that $\left\|\varphi_{\mathbf{u}}\right\|_{\mathbf{W}_{0}^{1, p^{\prime}}(\Omega)} \leq C_{2}$ for every $\mathbf{u} \in \mathcal{U}_{p}$. Using the expression for $F^{\prime \prime}$ given in (3.3), (4.7), and (4.10) we find for all $\mathbf{u} \in \mathcal{U}_{p}$

$$
\begin{aligned}
& \left|F^{\prime \prime}(\mathbf{u})(\mathbf{u}-\overline{\mathbf{u}})^{2}\right| \leq\left\|\mathbf{z}_{\mathbf{u}, \mathbf{u}-\overline{\mathbf{u}}}\right\|_{\mathbf{L}^{2}(\Omega)}^{2}+2\left\|\mathbf{z}_{\mathbf{u}, \mathbf{u}-\overline{\mathbf{u}}}\right\|_{\mathbf{L}^{2}(\Omega)}\left\|\mathbf{z}_{\mathbf{u}, \mathbf{u}-\overline{\mathbf{u}}}\right\|_{\mathbf{L}^{\frac{2 p}{2-p}}(\Omega)}\left\|\varphi_{\mathbf{u}}\right\|_{\mathbf{W}_{0}^{1, p^{\prime}}(\Omega)} \\
& \quad \leq\left(C_{\Omega, p}^{\prime}+2 C_{\Omega, p} C_{2}\right)\left\|\mathbf{z}_{\mathbf{u}, \mathbf{u}-\overline{\mathbf{u}}}\right\|_{\mathbf{W}_{0}^{1, p}(\Omega)}\left\|\mathbf{z}_{\mathbf{u}, \mathbf{u}-\overline{\mathbf{u}}}\right\|_{\mathbf{L}^{2}(\Omega)} \\
& \quad \leq\left(C_{\Omega, p}^{\prime}+2 C_{\Omega, p} C_{2}\right) M_{\mathcal{G}} M_{2}\|\mathbf{u}-\overline{\mathbf{u}}\|_{\mathbf{W}^{-1, p}(\Omega)}\left\|\mathbf{z}_{\mathbf{u}-\overline{\mathbf{u}}}\right\|_{\mathbf{L}^{2}(\Omega)} .
\end{aligned}
$$

We recall that the sufficient condition (4.5) is assumed and it involves two constants $\tau$ and $\delta$. Selecting

$$
0<\varepsilon_{2} \leq \min \left\{\frac{\tau}{\left(C_{\Omega, p}^{\prime}+2 C_{\Omega, p} C_{2}\right) M_{\mathcal{G}} M_{2}}, \frac{1}{M_{\mathcal{G}}}, \varepsilon_{1}\right\},
$$

where $\varepsilon_{1}$ was introduced in Lemma 4.6, we get

$$
\left|F^{\prime \prime}(\mathbf{u})(\mathbf{u}-\overline{\mathbf{u}})^{2}\right| \leq \tau\left\|\mathbf{z}_{\mathbf{u}-\overline{\mathbf{u}}}\right\|_{\mathbf{L}^{2}(\Omega)} \quad \forall \mathbf{u} \in B_{\varepsilon_{2}}(\overline{\mathbf{u}}) .
$$

Now, applying Lemma 4.8 with $\rho=\delta / 4$ we infer the existence of $\varepsilon \in\left(0, \varepsilon_{2}\right]$ such that

$$
\left|\left[F^{\prime \prime}(\mathbf{u})-F^{\prime \prime}(\overline{\mathbf{u}})\right](\mathbf{u}-\overline{\mathbf{u}})^{2}\right| \leq \frac{\delta}{2}\left\|\mathbf{z}_{\mathbf{u}-\overline{\mathbf{u}}}\right\|_{\mathbf{L}^{2}(\Omega)}^{2} \quad \forall \mathbf{u} \in B_{\varepsilon}(\overline{\mathbf{u}}) .
$$

Finally, we take

$$
\kappa=\min \left\{\frac{\delta}{2 M_{3}^{2}}, \frac{\tau}{M_{3}^{2}}\right\} .
$$

Now, we prove the inequality (4.6). To this end, we take $\mathbf{u} \in B_{\varepsilon}(\overline{\mathbf{u}})$. We distinguish two cases.

Case I. $\mathbf{u}-\overline{\mathbf{u}} \notin C_{\overline{\mathbf{u}}}^{\tau}$. In this case, we have

$$
F^{\prime}(\overline{\mathbf{u}})(\mathbf{u}-\overline{\mathbf{u}})+\alpha j^{\prime}(\overline{\mathbf{u}} ; \mathbf{u}-\overline{\mathbf{u}})>\tau\left\|\mathbf{z}_{\mathbf{u}-\overline{\mathbf{u}}}\right\|_{\mathbf{L}^{2}(\Omega)} .
$$

Due to the Lipschitz and convex properties of $j$ we also have

$$
j(\mathbf{u}) \geq j(\overline{\mathbf{u}})+j^{\prime}(\overline{\mathbf{u}} ; \mathbf{u}-\overline{\mathbf{u}}) .
$$

Additionally, from our selection of $\varepsilon$ we have with (4.7)

$$
\left\|\mathbf{z}_{\mathbf{u}-\overline{\mathbf{u}}}\right\|_{\mathbf{L}^{2}(\Omega)} \leq M_{\mathcal{G}}\|\mathbf{u}-\overline{\mathbf{u}}\|_{\mathbf{W}^{-1, p}(\Omega)} \leq M_{\mathcal{G}} \varepsilon \leq 1 .
$$

Using the last three inequalities, (4.20), (4.12), and making a Taylor expansion of $F$ around $\overline{\mathbf{u}}$ we get for some $\theta \in[0,1]$

$$
\begin{aligned}
& J(\mathbf{u}) \geq J(\overline{\mathbf{u}})+F^{\prime}(\overline{\mathbf{u}})(\mathbf{u}-\overline{\mathbf{u}})+\alpha j^{\prime}(\overline{\mathbf{u}} ; \mathbf{u}-\overline{\mathbf{u}})+\frac{1}{2} F^{\prime \prime}(\overline{\mathbf{u}}+\theta(\mathbf{u}-\overline{\mathbf{u}}))(\mathbf{u}-\overline{\mathbf{u}})^{2} \\
& \quad \geq J(\overline{\mathbf{u}})+\tau\left\|\mathbf{z}_{\mathbf{u}-\overline{\mathbf{u}}}\right\|_{\mathbf{L}^{2}(\Omega)}-\frac{\tau}{2}\left\|\mathbf{z}_{\mathbf{u}-\overline{\mathbf{u}}}\right\|_{\mathbf{L}^{2}(\Omega)}=J(\overline{\mathbf{u}})+\frac{\tau}{2}\left\|\mathbf{z}_{\mathbf{u}-\overline{\mathbf{u}}}\right\|_{\mathbf{L}^{2}(\Omega)} \\
& \quad \geq J(\overline{\mathbf{u}})+\frac{\tau}{2}\left\|\mathbf{z}_{\mathbf{u}-\overline{\mathbf{u}}}\right\|_{\mathbf{L}^{2}(\Omega)}^{2} \geq J(\overline{\mathbf{u}})+\frac{\tau}{2 M_{3}^{2}}\left\|\mathbf{y}_{\mathbf{u}}-\overline{\mathbf{y}}\right\|_{\mathbf{L}^{2}(\Omega)}^{2} \geq J(\overline{\mathbf{u}})+\frac{\kappa}{2}\left\|\mathbf{y}_{\mathbf{u}}-\overline{\mathbf{y}}\right\|_{\mathbf{L}^{2}(\Omega)}^{2}
\end{aligned}
$$

Copyright $@$ ㅇ by SIAM. Unauthorized reproduction of this article is prohibited. 
Case II. $\mathbf{u}-\overline{\mathbf{u}} \in C_{\overline{\mathbf{u}}}^{\tau}$. We proceed similarly to the previous case and use (4.1), (4.5), (4.21), and (4.12):

$$
\begin{aligned}
J(\mathbf{u}) & \geq J(\overline{\mathbf{u}})+F^{\prime}(\overline{\mathbf{u}})(\mathbf{u}-\overline{\mathbf{u}})+\alpha j^{\prime}(\overline{\mathbf{u}} ; \mathbf{u}-\overline{\mathbf{u}})+\frac{1}{2} F^{\prime \prime}(\overline{\mathbf{u}}+\theta(\mathbf{u}-\overline{\mathbf{u}}))(\mathbf{u}-\overline{\mathbf{u}})^{2} \\
& \geq J(\overline{\mathbf{u}})+\frac{1}{2} F^{\prime \prime}(\overline{\mathbf{u}})(\mathbf{u}-\overline{\mathbf{u}})^{2}+\frac{1}{2}\left[F^{\prime \prime}(\overline{\mathbf{u}}+\theta(\mathbf{u}-\overline{\mathbf{u}}))-F^{\prime \prime}(\overline{\mathbf{u}})\right](\mathbf{u}-\overline{\mathbf{u}})^{2} \\
& \geq J(\overline{\mathbf{u}})+\frac{\delta}{2}\left\|\mathbf{z}_{\mathbf{u}-\overline{\mathbf{u}}}\right\|_{\mathbf{L}^{2}(\Omega)}^{2}-\frac{\delta}{4}\left\|\mathbf{z}_{\mathbf{u}-\overline{\mathbf{u}}}\right\|_{\mathbf{L}^{2}(\Omega)}^{2}=J(\overline{\mathbf{u}})+\frac{\delta}{4}\left\|\mathbf{z}_{\mathbf{u}-\overline{\mathbf{u}}}\right\|_{\mathbf{L}^{2}(\Omega)}^{2} \\
& \geq J(\overline{\mathbf{u}})+\frac{\delta}{4 M_{3}^{2}}\left\|\mathbf{y}_{\mathbf{u}}-\overline{\mathbf{y}}\right\|_{\mathbf{L}^{2}(\Omega)}^{2} \geq J(\overline{\mathbf{u}})+\frac{\kappa}{2}\left\|\mathbf{y}_{\mathbf{u}}-\overline{\mathbf{y}}\right\|_{\mathbf{L}^{2}(\Omega)}^{2} .
\end{aligned}
$$

5. Stability of the optimal states with respect to perturbations of the data of $(\mathrm{P})$. In this section we assume that $(\overline{\mathbf{y}}, \overline{\mathbf{u}})$ is a strict local solution of $(\mathrm{P})$ in the $\mathbf{W}_{0}^{1, p}(\Omega) \times \mathbf{W}^{-1, p}(\Omega)$ topology such that $\overline{\mathbf{y}}$ is regular in the sense of Definition 2.7. Hence, we can formulate the control problem $\left(\mathrm{P}_{\text {loc }}\right)$ as in section 3 , choosing $\mathcal{U}_{p}$ small enough so that $\overline{\mathbf{u}}$ is the unique global minimizer of $J$ in $\mathcal{U}_{p}$. We recall that, as proved in section 3 , for every $\mathbf{u} \in \mathcal{U}_{p}$ there exists a unique solution $\mathbf{y}_{\mathbf{u}} \in \mathcal{Y}_{p}$ of (1.1).

We are going to prove the stability of $\overline{\mathbf{y}}$ with respect to small perturbations in some data of the control problem $\left(\mathrm{P}_{\text {loc }}\right)$. For this analysis the growth condition (4.6) will play a key role. We recall that (4.6) is deduced from the sufficient second order condition (4.5). The fact that $B_{\varepsilon}(\overline{\mathbf{u}})$ in (4.6) is a ball in $\mathbf{W}^{-1, p}(\Omega)$ rather than in $\mathbf{M}(\omega)$ is essential to prove the stability results.

We will consider two kinds of perturbations in $\left(\mathrm{P}_{\mathrm{loc}}\right)$ : perturbations in the forcing $\mathbf{f}_{0}$ and in the observation $\mathbf{y}_{d}$.

5.1. Perturbations in the forcing. Here we consider the following control problems

$$
\left(\mathrm{P}_{\rho}\right) \quad \min _{\mathbf{u} \in \mathcal{U}_{p}} J_{\rho}(\mathbf{u})=\frac{1}{2} \int_{\Omega}\left|\mathbf{y}_{\rho, \mathbf{u}}-\mathbf{y}_{d}\right|^{2} d x+\alpha\|\mathbf{u}\|_{\mathbf{M}(\omega)},
$$

$\mathbf{y}_{\rho, \mathbf{u}}$ being the unique solution in $\mathcal{Y}_{p}$ of the perturbed system

$$
\left\{\begin{array}{l}
-\nu \Delta \mathbf{y}+(\mathbf{y} \cdot \nabla) \mathbf{y}+\nabla \mathfrak{p}=\mathbf{f}_{\rho}+\chi_{\omega} \mathbf{u} \text { in } \Omega, \\
\operatorname{div} \mathbf{y}=0 \text { in } \Omega, \mathbf{y}=0 \text { on } \Gamma,
\end{array}\right.
$$

where $\mathbf{f}_{\rho} \in \mathbf{W}^{-1, p}(\Omega)$ satisfies

$$
\left\|\mathbf{f}_{0}-\mathbf{f}_{\rho}\right\|_{\mathbf{W}^{-1, p}(\Omega)} \leq C_{0} \rho
$$

with $C_{0}$ independent of $\rho$. From Theorem 2.10 we know that (5.1) has a unique solution $\mathbf{y}_{\rho, \mathbf{u}} \in \mathcal{Y}_{p}$ if $\rho$ is small enough. Moreover, using the mean value theorem and (4.7) we have

$$
\begin{aligned}
& \left\|\mathbf{y}_{\rho, \mathbf{u}}-\mathbf{y}_{\mathbf{u}}\right\|_{\mathbf{W}_{0}^{1, p}(\Omega)}=\left\|\mathcal{G}\left(\mathbf{f}_{\rho}+\chi_{\omega} \mathbf{u}\right)-\mathcal{G}\left(\mathbf{f}_{0}+\chi_{\omega} \mathbf{u}\right)\right\|_{\mathbf{W}_{0}^{1, p}(\Omega)} \\
& \quad \leq M_{\mathcal{G}}\left\|\mathbf{f}_{\rho}-\mathbf{f}_{0}\right\|_{\mathbf{W}^{-1, p}(\Omega)} \leq M_{\mathcal{G}} C_{0} \rho .
\end{aligned}
$$

Using this property, by classical arguments (see, for instance, [7]), one can prove the existence of $\hat{\rho}>0$ and an open neighborhood $\hat{\mathcal{U}}_{p} \subset \mathcal{U}_{p}$ of $\overline{\mathbf{u}}$ such that for every $\rho \in(0, \hat{\rho})$ the control problem $\left(\mathrm{P}_{\rho}\right)$ has a global minimizer $\overline{\mathbf{u}}_{\rho}$ in $\hat{\mathcal{U}}_{p}$. Moreover, it holds when $\rho \rightarrow 0$ 


$$
\begin{gathered}
\overline{\mathbf{u}}_{\rho} \stackrel{*}{\rightarrow} \overline{\mathbf{u}} \text { in } \mathbf{M}(\omega), \\
\left(\overline{\mathbf{y}}_{\rho}, \overline{\mathbf{u}}_{\rho}\right) \rightarrow(\overline{\mathbf{y}}, \overline{\mathbf{u}}) \text { strongly in } \mathbf{W}_{0}^{1, p}(\Omega) \times \mathbf{W}^{-1, p}(\Omega),
\end{gathered}
$$

where $\overline{\mathbf{y}}_{\rho}=\overline{\mathbf{y}}_{\rho, \overline{\mathbf{u}}_{\rho}}$.

The next theorem establishes a rate of the convergence result for $\overline{\mathbf{y}}_{\rho}$ to $\overline{\mathbf{y}}$.

THEOREM 5.1. Let us assume that $\overline{\mathbf{u}}$ satisfies (4.6); then there exist $\rho_{0} \in(0, \hat{\rho})$ and a constant $K>0$ such that

$$
\left\|\overline{\mathbf{y}}_{\rho}-\overline{\mathbf{y}}\right\|_{\mathbf{L}^{2}(\Omega)} \leq K \sqrt{\rho} \quad \forall \rho \leq \rho_{0} .
$$

Proof. Due to (5.5), there exists $\rho_{0} \in(0, \hat{\rho})$ such that $\overline{\mathbf{u}}_{\rho} \in B_{\varepsilon}(\overline{\mathbf{u}})$ for every $\rho \leq \rho_{0}$. Let us introduce the functions $\mathbf{y}_{\rho, \overline{\mathbf{u}}}$ and $\mathbf{y}_{\overline{\mathbf{u}}_{\rho}}$ solutions of (5.1) and (1.1) corresponding to the controls $\overline{\mathbf{u}}$ and $\overline{\mathbf{u}}_{\rho}$, respectively. Using that $\overline{\mathbf{u}}_{\rho}$ is a global minimizer of $J_{\rho}$ in $\hat{\mathcal{U}}_{p}$ and (4.6) we infer

$$
\begin{aligned}
& J_{\rho}(\overline{\mathbf{u}}) \geq J_{\rho}\left(\overline{\mathbf{u}}_{\rho}\right)=J\left(\overline{\mathbf{u}}_{\rho}\right)+\left[J_{\rho}\left(\overline{\mathbf{u}}_{\rho}\right)-J\left(\overline{\mathbf{u}}_{\rho}\right)\right] \\
& \geq J(\overline{\mathbf{u}})+\frac{\kappa}{2}\left\|\mathbf{y}_{\overline{\mathbf{u}}_{\rho}}-\overline{\mathbf{y}}\right\|_{\mathbf{L}^{2}(\Omega)}^{2}+\left[J_{\rho}\left(\overline{\mathbf{u}}_{\rho}\right)-J\left(\overline{\mathbf{u}}_{\rho}\right)\right] .
\end{aligned}
$$

From this inequality we deduce

$$
\begin{aligned}
& \kappa\left\|\mathbf{y}_{\overline{\mathbf{u}}_{\rho}}-\overline{\mathbf{y}}\right\|_{\mathbf{L}^{2}(\Omega)}^{2} \leq 2\left[J_{\rho}(\overline{\mathbf{u}})-J(\overline{\mathbf{u}})\right]-2\left[J_{\rho}\left(\overline{\mathbf{u}}_{\rho}\right)-J\left(\overline{\mathbf{u}}_{\rho}\right)\right] \\
& \quad=\int_{\Omega}\left[\left|\mathbf{y}_{\rho, \overline{\mathbf{u}}}-\mathbf{y}_{d}\right|^{2}-\left|\overline{\mathbf{y}}-\mathbf{y}_{d}\right|^{2}\right] d x-\int_{\Omega}\left[\left|\overline{\mathbf{y}}_{\rho}-\mathbf{y}_{d}\right|^{2}-\left|\mathbf{y}_{\overline{\mathbf{u}}_{\rho}}-\mathbf{y}_{d}\right|^{2}\right] d x \\
& \quad \leq\left\|\mathbf{y}_{\rho, \overline{\mathbf{u}}}+\overline{\mathbf{y}}-2 \mathbf{y}_{d}\right\|_{\mathbf{L}^{2}(\Omega)}\left\|\mathbf{y}_{\rho, \overline{\mathbf{u}}}-\overline{\mathbf{y}}\right\|_{\mathbf{L}^{2}(\Omega)}+\left\|\overline{\mathbf{y}}_{\rho}+\mathbf{y}_{\overline{\mathbf{u}}_{\rho}}-2 \mathbf{y}_{d}\right\|_{\mathbf{L}^{2}(\Omega)}\left\|\overline{\mathbf{y}}_{\rho}-\mathbf{y}_{\overline{\mathbf{u}}_{\rho}}\right\|_{\mathbf{L}^{2}(\Omega)} .
\end{aligned}
$$

Let us observe that taking $\mathbf{u}=\overline{\mathbf{u}}$ in (5.3) we deduce the convergence $\mathbf{y}_{\rho, \overline{\mathbf{u}}} \rightarrow \overline{\mathbf{y}}$ in $\mathbf{W}_{0}^{1, p}(\Omega)$. Moreover, by (5.5) $\mathbf{y}_{\overline{\mathbf{u}}_{\rho}}=\mathcal{G}\left(\mathbf{f}_{\rho}+\chi_{\omega} \overline{\mathbf{u}}_{\rho}\right) \rightarrow \mathcal{G}\left(\mathbf{f}_{0}+\chi_{\omega} \overline{\mathbf{u}}\right)=\bar{y}$ in $\mathbf{W}_{0}^{1, p}(\Omega)$ holds. Therefore, the sequences $\left\{\mathbf{y}_{\rho, \overline{\mathbf{u}}}\right\}_{\rho \leq \rho_{0}}$ and $\left\{\mathbf{y}_{\overline{\mathbf{u}}_{\rho}}\right\}_{\rho \leq \rho_{0}}$ are bounded in $\mathbf{W}_{0}^{1, p}(\Omega)$. The boundedness of the sequence $\left\{\overline{\mathbf{y}}_{\rho}\right\}_{\rho \leq \rho_{0}}$ follows from (5.5). Taking into account these facts and using twice (5.3) with $\mathbf{u}=\overline{\mathbf{u}}$ and $\mathbf{u}=\overline{\mathbf{u}}_{\rho}$, we obtain from the above inequality

$$
\left\|\mathbf{y}_{\overline{\mathbf{u}}_{\rho}}-\overline{\mathbf{y}}\right\|_{\mathbf{L}^{2}(\Omega)} \leq C \sqrt{\rho} \quad \forall \rho \leq \rho_{0} .
$$

Finally, from (5.3) with $\mathbf{u}=\overline{\mathbf{u}}_{\rho}$ and (5.7) we conclude

$$
\left\|\overline{\mathbf{y}}_{\rho}-\overline{\mathbf{y}}\right\|_{\mathbf{L}^{2}(\Omega)} \leq\left\|\overline{\mathbf{y}}_{\rho}-\mathbf{y}_{\overline{\mathbf{u}}_{\rho}}\right\|_{\mathbf{L}^{2}(\Omega)}+\left\|\mathbf{y}_{\overline{\mathbf{u}}_{\rho}}-\overline{\mathbf{y}}\right\|_{\mathbf{L}^{2}(\Omega)} \leq C_{1} \rho+C \sqrt{\rho},
$$

which proves (5.6).

While we cannot expect to obtain a rate of convergence for $\left\|\overline{\mathbf{u}}_{\rho}-\mathbf{u}\right\|_{\mathbf{M}(\omega)}$ we still have the following corollary to the previous theorem.

COROLlaRY 5.2. There exists $K^{\prime}>0$ such that $\left|\left\|\overline{\mathbf{u}}_{\rho}\right\|_{\mathbf{M}(\omega)}-\|\mathbf{u}\|_{\mathbf{M}(\omega)}\right| \leq K^{\prime} \sqrt{\rho}$ for all $\rho \leq \rho_{0}$.

Proof. From the inequality $J(\overline{\mathbf{u}}) \leq J\left(\overline{\mathbf{u}}_{\rho}\right)$ we get with (5.7)

$$
\begin{aligned}
& \alpha\left(\|\overline{\mathbf{u}}\|_{\mathbf{M}(\omega)}-\left\|\overline{\mathbf{u}}_{\rho}\right\|_{\mathbf{M}(\omega)}\right) \leq \frac{1}{2}\left[\left\|\mathbf{y}_{\overline{\mathbf{u}}_{\rho}}-\mathbf{y}_{d}\right\|_{\mathbf{L}^{2}(\Omega)}^{2}-\left\|\overline{\mathbf{y}}-\mathbf{y}_{d}\right\|_{\mathbf{L}^{2}(\Omega)}^{2}\right] \\
& \quad \leq C_{1}\left\|\mathbf{y}_{\overline{\mathbf{u}}_{\rho}}-\overline{\mathbf{y}}\right\|_{\mathbf{L}^{2}(\Omega)} \leq C_{1} C \sqrt{\rho} .
\end{aligned}
$$

Copyright $@$ by SIAM. Unauthorized reproduction of this article is prohibited. 
Analogously, using that $J_{\rho}\left(\overline{\mathbf{u}}_{\rho}\right) \leq J_{\rho}(\overline{\mathbf{u}})$ and estimates (5.3) and (5.7) we obtain

$$
\begin{aligned}
& \alpha\left(\left\|\overline{\mathbf{u}}_{\rho}\right\|_{\mathbf{M}(\omega)}-\|\overline{\mathbf{u}}\|_{\mathbf{M}(\omega)}\right) \leq \frac{1}{2}\left[\left\|\mathbf{y}_{\rho, \overline{\mathbf{u}}}-\mathbf{y}_{d}\right\|_{\mathbf{L}^{2}(\Omega)}^{2}-\left\|\overline{\mathbf{y}}_{\rho}-\mathbf{y}_{d}\right\|_{\mathbf{L}^{2}(\Omega)}^{2}\right] \\
& \leq C_{2}\left\|\mathbf{y}_{\rho, \overline{\mathbf{u}}}-\overline{\mathbf{y}}_{\rho}\right\|_{\mathbf{L}^{2}(\Omega)} \leq C_{2}\left(\left\|\mathbf{y}_{\rho, \overline{\mathbf{u}}}-\overline{\mathbf{y}}\right\|_{\mathbf{L}^{2}(\Omega)}+\left\|\overline{\mathbf{y}}-\overline{\mathbf{y}}_{\rho}\right\|_{\mathbf{L}^{2}(\Omega)}\right) \leq C_{3} \sqrt{\rho} .
\end{aligned}
$$

Combining (5.8) and (5.9) the corollary follows.

5.2. Perturbations in the observation. In this section we consider perturbations of the observation $\mathbf{y}_{d}$. We formulate the following control problems

$$
\left(\mathrm{P}_{\rho}\right) \quad \min _{\mathbf{u} \in \mathcal{U}_{p}} J_{\rho}(\mathbf{u})=\frac{1}{2} \int_{\Omega}\left|\mathbf{y}_{\mathbf{u}}-\mathbf{y}_{d}^{\rho}\right|^{2} d x+\alpha\|\mathbf{u}\|_{\mathbf{M}(\omega)},
$$

where $\mathbf{y}_{\mathbf{u}}$ denotes the solution of (1.1) corresponding to the control $\mathbf{u}$ and $\mathbf{y}_{d}^{\rho} \in \mathbf{L}^{2}(\Omega)$ is a perturbation of $\mathbf{y}_{d}$ satisfying

$$
\left\|\mathbf{y}_{d}^{\rho}-\mathbf{y}_{d}\right\|_{\mathbf{L}^{2}(\Omega)} \leq C_{0} \rho .
$$

With the notation of the previous subsection and using the arguments of [7], the existence of $\left(\overline{\mathbf{y}}_{\rho}, \overline{\mathbf{u}}_{\rho}\right)$ and the convergence properties (5.4)-(5.5) follow. Observe that now $\overline{\mathbf{y}}_{\rho}=\mathbf{y}_{\overline{\mathbf{u}}_{\rho}}$ is the solution of (1.1) associated with $\overline{\mathbf{u}}_{\rho}$. Concerning the convergence rate for the optimal states we have the following result.

Theorem 5.3. Let us assume that $\overline{\mathbf{u}}$ satisfies (4.6). Then there exist $\rho_{0} \in(0, \hat{\rho})$ and a constant $K>0$ such that

$$
\left\|\overline{\mathbf{y}}_{\rho}-\overline{\mathbf{y}}\right\|_{\mathbf{L}^{2}(\Omega)} \leq K \rho \quad \forall \rho \leq \rho_{0} .
$$

Proof. Once again, due to (5.5), there exists $\rho_{0} \in(0, \hat{\rho})$ such that $\overline{\mathbf{u}}_{\rho} \in \bar{B}_{\varepsilon}(\overline{\mathbf{u}})$ for every $\rho \leq \rho_{0}$. Then, we get

$$
\begin{aligned}
& J_{\rho}(\overline{\mathbf{u}}) \geq J_{\rho}\left(\overline{\mathbf{u}}_{\rho}\right)=J\left(\overline{\mathbf{u}}_{\rho}\right)+\left[J_{\rho}\left(\overline{\mathbf{u}}_{\rho}\right)-J\left(\overline{\mathbf{u}}_{\rho}\right)\right] \\
& \quad \geq J(\overline{\mathbf{u}})+\frac{\kappa}{2} \| \overline{\mathbf{y}}_{\rho}-\overline{\mathbf{y}}_{\mathbf{L}^{2}(\Omega)}^{2}+\left[J_{\rho}\left(\overline{\mathbf{u}}_{\rho}\right)-J\left(\overline{\mathbf{u}}_{\rho}\right)\right] .
\end{aligned}
$$

Rearranging terms we get

$$
\begin{aligned}
\kappa\left\|\overline{\mathbf{y}}_{\rho}-\overline{\mathbf{y}}\right\|_{\mathbf{L}^{2}(\Omega)}^{2} & \\
\quad & \int_{\Omega}\left[\left|\overline{\mathbf{y}}-\mathbf{y}_{d}^{\rho}\right|^{2}-\left|\overline{\mathbf{y}}-\mathbf{y}_{d}\right|^{2}\right] d x-\int_{\Omega}\left[\left|\overline{\mathbf{y}}_{\rho}-\mathbf{y}_{d}^{\rho}\right|^{2}-\left|\overline{\mathbf{y}}_{\rho}-\mathbf{y}_{d}\right|^{2}\right] d x \\
& =\int_{\Omega}\left(2 \overline{\mathbf{y}}-\mathbf{y}_{d}-\mathbf{y}_{d}^{\rho}\right) \cdot\left(\mathbf{y}_{d}-\mathbf{y}_{d}^{\rho}\right) d x-\int_{\Omega}\left(2 \overline{\mathbf{y}}_{\rho}-\mathbf{y}_{d}^{\rho}-\mathbf{y}_{d}\right) \cdot\left(\mathbf{y}_{d}-\mathbf{y}_{d}^{\rho}\right) d x \\
& =2 \int_{\Omega}\left(\overline{\mathbf{y}}-\overline{\mathbf{y}}_{\rho}\right) \cdot\left(\mathbf{y}_{d}-\mathbf{y}_{d}^{\rho}\right) d x \\
& \leq 2\left\|\overline{\mathbf{y}}-\overline{\mathbf{y}}_{\rho}\right\|_{\mathbf{L}^{2}(\Omega)}\left\|\mathbf{y}_{d}-\mathbf{y}_{d}^{\rho}\right\|_{\mathbf{L}^{2}(\Omega)} \leq 2 C_{0} \rho\left\|\overline{\mathbf{y}}-\overline{\mathbf{y}}_{\rho}\right\|_{\mathbf{L}^{2}(\Omega)},
\end{aligned}
$$

which leads to (5.11).

Analogous to Corollary 5.2 we easily obtain the following consequence of the above theorem.

Corollary 5.4. There exists $K^{\prime}>0$ such that $\left|\left\|\overline{\mathbf{u}}_{\rho}\right\|_{\mathbf{M}(\omega)}-\|\mathbf{u}\|_{\mathbf{M}(\omega)}\right| \leq K^{\prime} \rho$ for all $\rho \leq \rho_{0}$.

Copyright (c) by SIAM. Unauthorized reproduction of this article is prohibited. 
Proof. It is enough to observe that

$$
J_{\rho}\left(\overline{\mathbf{u}}_{\rho}\right)-J\left(\overline{\mathbf{u}}_{\rho}\right) \leq J_{\rho}\left(\overline{\mathbf{u}}_{\rho}\right)-J(\overline{\mathbf{u}}) \leq J_{\rho}(\overline{\mathbf{u}})-J(\overline{\mathbf{u}})
$$

and to use (5.10) and (5.11).

\section{REFERENCES}

[1] E. CASAS, A review on sparse solutions in optimal control of partial differential equations, SEMA Journal, 74 (2017), pp. 319-344.

[2] E. Casas, C. Clason, and K. Kunisch, Approximation of elliptic control problems in measure spaces with sparse solutions, SIAM J. Control Optim., 50 (2012), pp. 1735-1752.

[3] E. Casas, C. Clason, and K. Kunisch, Parabolic control problems in measure spaces with sparse solutions, SIAM J. Control Optim., 51 (2013), pp. 28-63.

[4] E. CASAS AND K. KUnisch, Optimal control of semilinear elliptic equations in measure spaces, SIAM J. Control Optim., 52 (2014), pp. 339-364.

[5] E. Casas and K. Kunisch, Parabolic control problems in space-time measure spaces, ESAIM Control Optim. Calc. Var., 22 (2016), pp. 355-370.

[6] E. Casas, M. Mateos, AND J.-P. Raymond, Error estimates for the numerical approximation of a distributed control problem for the steady-state Navier-Stokes equations, SIAM J. Control Optim., 46 (2007), pp. 952-982.

[7] E. Casas and F. Tröltzsch, Second order optimality conditions for weak and strong local solutions of parabolic optimal control problems, Vietnam J. Math., 44 (2016), pp. 181-202.

[8] E. Casas, B. Vexler, and E. Zuazua, Sparse initial data identification for parabolic PDE and its finite element approximations, Math. Control Relat. Fields, 5 (2015), pp. 377-399.

[9] E. Casas and E. Zuazua, Spike controls for elliptic and parabolic PDEs, Systems Control Lett., 62 (2013), pp. 311-318.

[10] C. Clason and K. Kunisch, A duality-based approach to elliptic control problems in nonreflexive Banach spaces, ESAIM Control Optim. Calc. Var., 17 (2011), pp. 243-266.

[11] C. Clason and K. Kunisch, A measure space approach to optimal source placement, Comput. Optim. Appl., 53 (2012), pp. 155-171.

[12] J. De LOs REYES AND R. GRIESSE, State-constrained optimal control of the three-dimensional stationary Navier-Stokes equations, J. Math. Anal. Appl., 343 (2008), pp. 257-272.

[13] M. Desai And K. Ito, Optimal controls of Navier-Stokes equations, SIAM J. Control Optim., 5 (1994), pp. 1428-1446.

[14] J. DunN, On second order sufficient optimality conditions for structured nonlinear programs in infinite-dimensional function spaces, in Mathematical Programming with Data Perturbations, A. Fiacco, ed., Marcel Dekker, New York, 1998, pp. 83-107.

[15] G. GaLdI, An Introduction to the Mathematical Theory of the Navier-Stokes Equations, Springer, New York, 2011.

[16] P. Girault and P. Raviart, Finite Element Methods for Navier-Stokes Equations. Theory and Algorithms, Springer-Verlag, New York, 1986.

[17] M. Gunzburger, Flow Control, Springer-Verlag, New York, 1995.

[18] H. KIM, Existence and regularity of very weak solutions of the stationary Navier-Stokes equations, Arch. Rational Mech. Anal., 193 (2009), pp. 117-152.

[19] K. Kunisch, K. Pieper, And B. Vexler, Measure valued directional sparsity for parabolic optimal control problems, SIAM J. Control Optim., 52 (2014), pp. 3078-3108.

[20] K. Kunisch, P. Trautmann, and B. Vexler, Optimal control of the undamped linear wave equation with measure valued controls, SIAM J. Control Optim., 54 (2016), pp. 1212-1244.

[21] H. MAURER AND J. Zowe, First and second order necessary and sufficient optimality conditions for infinite-dimensional programming problems, Math. Program., 16 (1979), pp. 98-110.

[22] J. NeČAs, Les Méthodes Directes en Théorie des Equations Elliptiques, Editeurs Academia, 1967.

[23] W. Rudin, Real and Complex Analysis, McGraw-Hill Book, London, 1970.

[24] D. Serre, Les équations de Navier-Stokes stationnaries avec données peu reguliéres, Ann. Sci. Norm. Sup. Pisa, 10 (1983), pp. 543-559.

[25] G. StAdLER, Elliptic optimal control problems with $L^{1}$-control cost and applications for the placement of control devices, Comput. Optim. Appl., 44 (2009), pp. 159-181.

[26] F. Tröltzsch and D. Wachsmuth, Second-order sufficient optimality conditions for the optimal control of Navier-Stokes equations, ESAIM Control Optim. Calc. Var., 12 (2006), pp. $93-119$

Copyright (C) by SIAM. Unauthorized reproduction of this article is prohibited. 